\title{
Conformations of the Pyranoid Sugars. II. Infrared Absorption Spectra of Some Aldopyranosides
}

\author{
R. Stuart Tipson and Horace S. Isbell
}

(January 29, 1960)

\begin{abstract}
The conformations of twenty-four aldopyranosides have been studied by analysis of their infrared absorption spectra. The most stable conformations of twelve of the glycosides had previously been assigned by Reeves from a study of their instability factors; these conformations were assumed to apply to the crystalline state, for which the spectra had been recorded. The compounds were classified into (a) configurationally and (b) structurally related groups, and the spectra were intercompared. The analysis revealed groups of absorption bands which showed a concerted shift on change of anomeric disposition.

With these groups of absorption bands thus identified, intercomparison with nine of the remaining spectra afforded evidence that the anomeric group (1) is axial in methyl D-glycero- $\alpha$-L-gluco-heptopyranoside, methyl D-glycero- $\alpha$-L-manno-heptopyranoside, and methyl D-glycero- $\alpha$-D-gulo-heptopyranoside; (2) is equatorial in methyl 6 -deoxy- $\beta$-L-mannopyranoside, methyl D-glycero- $\beta$-D-gulo-heptopyranoside, and cyclohexyl D-glycero- $\beta$-D-gulo-heptopyranoside; and (3) either is quasi or occurs as different (or mixed) axial and equatorial forms in methyl $\alpha$-D-lyxopranoside, methyl $\beta$-D-lyxopranoside, and (possibly) $\alpha$-D-methylgulopyranoside.

Three of the glycosides were available as their crystalline complexes with calcium chloride. The spectra of these complexes were also examined, and the effect of co-crystallization with calcium chloride is pointed out.
\end{abstract}

\section{Scope and Purpose of the Project}

The shape or conformation of a molecule greatly influences its rates of reaction and other properties. For this reason, detailed knowledge of the conformations of pyranoid derivatives is desirable. Our prior publications on this subject have presented a system for naming the conformations of pyranoid compounds $[1,2]{ }^{1}$

The conformations of numerous methyl glycosides and other pyranoid derivatives have been determined by Reeves from (a) a study of stereomeric factors and (b) the type of complex formed in cuprammonia solution. Reaction of cuprammonia with an aldopyranoside may sometimes cause alteration in the conformation. Hence, assignments of conformation based on the formation of copper complexes need confirmation by measurement of at least one relevant physical characteristic.

The work herein reported was primarily undertaken to provide infrared spectrograms of aldosides having the pyranoid ring, with the object of discovering correlations that might be of value in conformational analysis. It seemed possible that the axial or equatorial disposition of reference groups in the various molecules of glycopyranosides should give rise to different vibrations, and that it should, accordingly, be feasible to identify certain bands in their spectra as being characteristic of the different ways of arranging the reference groups. The infrared spectra for a group of aldopyranosides have, there-

1 Figures in brackets indicate the literature references at the end of this paper. The references for table 1 are given at the end of the table. fore, been recorded, and the bands have been compiled and then studied by statistical and comparative methods.

Prior publications from our laboratory on related topics have dealt with the infrared absorption spectra of sugar acetates [3] and of some cyclic acetals of sugars [4], and with a system for classifying carbohydrate derivatives for comparative purposes [5]. The acetals previously studied are polycyclic and have fused or bridged rings; the conformations of such molecules are "locked." On the other hand, the pyranoid ring of some unsubstituted glycopyranosides is flexible, and the conformation adopted may depend on the physical conditions; thus, the conformation of the molecules in solution, particularly in the presence of a complexing agent, may be different from that of the molecules in the crystalline material. In the present study, the infrared absorption spectra of compounds in the solid phase have been recorded, and a method has been developed for assignment of conformation from analysis of the infrared absorption spectra and comparison with the spectra of glycosides of known conformation. ${ }^{2}$

\section{Compounds Investigated}

Table 1 gives a list of the compounds, their code numbers $[5],{ }^{3}$ predicted stable conformations, and an index to the spectrograms; the serial number of a compound is the same as the number of its spectro-

\footnotetext{
2 The method of comparison is being applied to other groups of structurally related pyranoid sugar derivatives.

3 Code numbers are assigned according to a previously published classification. system [5], for use with punched-card techniques.
} 
gram. The spectra were measured in the region of 5000 to $667 \mathrm{~cm}^{-1}$ (sodium chloride optics) and in the region of 667 to $250 \mathrm{~cm}^{-1}$ (cesium bromide optics). The spectrograms are given together with a discussion of (a) the structure of the compounds and (b) some of the outstanding features of their spectra.

All of the compounds listed in table 1 are glycosides of aldoses, and all have the pyranoid ring. As a common structural feature, all but one of the glycosides have a glycosidic methoxyl group; one has a glycosidic cyclohexyloxy group. The glycosides differ in regard to one or more of the following features: (a) the $\alpha$ or $\beta$ anomeric configuration at carbon atom 1, (b) the configurations of the other carbon atoms of the pyranoid ring, (c) the nature and configuration of the substituent, if any, at carbon atom 5 of the pyranoid ring, and (d) the configuration of carbon atom 5 in those glycosides in which this atom is asvmmetric.

The conformation selected by Reeves as probably the most stable is shown in table 1 for each of those glycosides mentioned that he studied. The conformations are indicated by the system devised by Isbell and Tipson $[1,2]$; the symbol CA means "that chair conformation for which the $\alpha$ anomeric group is axial," and the symbol CE means "that chair conformation for which the $\alpha$ anomeric group is equatorial." The chair forms and nomenclature for the anomers of the methyl aldopyranosides are shown in figures 1 and 2 . The infrared absorption spectra of the same polymorphic modification of two members of an enantiomorphic pair are indistinguishable.
Hence, in the present study, the spectrum of whichever member was available was examined, and was considered to apply to the other enantiomorph.

\section{Reference Aldopyranosides of Known Conformation}

Each methyl aldopyranoside is theoretically capable of assuming at least one of a variety of different conformations, depending on the conditions. From studies made by Reeves [6 to 8], it would appear that two groups (A and B) of methyl aldopyranosides can be distinguished. Glycosides in group A have one of the two chair-forms. The members of group B exist either (a) as a mixture of the two chair-forms or (b) as some other conformation.

It seemed likely that, in the solid state, each individual aldopyranoside would exist in only one conformation; and this conformation would presumably be the one it took as it crystallized from solution. As a working hypothesis, it was assumed that the conformation of an aldopyranoside in the crystalline state is the same as the stable form predicted by Reeves. It would then be reasonable to expect that examination of the infrared absorption spectra of the solid phase of those glycopyranosides of group A (each believed to assume a single chair-conformation), followed by a comparison with the spectra of the other group (B) of aldopyranosides, might provide evidence regarding the conformations (in the solid phase) of the members of the latter group (B).

TABLE 1. Compounds measured, stable conformations, and index to spectrograms

\begin{tabular}{|c|c|c|c|c|c|c|c|c|}
\hline \multirow[b]{2}{*}{ Code a } & \multirow[b]{2}{*}{ Compound } & \multirow[b]{2}{*}{ Reference } & \multicolumn{5}{|c|}{ Stable conformation b } & \multirow{2}{*}{$\begin{array}{l}\text { Spectro- } \\
\text { gram }\end{array}$} \\
\hline & & & $\begin{array}{c}\text { Reeves' } \\
\text { assignment c }\end{array}$ & $\begin{array}{l}\text { Anomeric } \\
\text { disposition }\end{array}$ & Reference & $\begin{array}{l}\text { Present } \\
\text { assignment d }\end{array}$ & $\begin{array}{l}\text { Anomeric } \\
\text { disposition d }\end{array}$ & \\
\hline $\begin{array}{l}\text { 10. } 11111 \\
10.11211 \\
\text { 10. } 21111 \\
\text { 10. } 21211 \\
10.41111\end{array}$ & $\begin{array}{l}\text { Methyl } \alpha \text {-D-xylopyranoside } \\
\text { Methyl } \beta \text {-D-xylopyranoside } \\
\text { Methyl } \alpha \text {-D-glucopyranoside } \\
\text { Methyl } \beta \text {-D-glucopyranoside } \\
\text { Methyl D-glycero- } \alpha \text {-L-gluco-heptopyranoside }\end{array}$ & $\begin{array}{r}1,2 \\
1,2 \\
3 \\
3 \\
4\end{array}$ & $\begin{array}{l}\mathrm{CA} \\
\mathrm{CA} \\
\mathrm{CA} \\
\mathrm{CA}\end{array}$ & $e e_{\ldots} \ldots \ldots$ & $\begin{array}{l}13 \\
13 \\
14 \\
14\end{array}$ & $\begin{array}{l}\mathrm{CA} \\
\mathrm{CA} \\
\mathrm{CA} \\
\mathrm{CA}\end{array}$ & $\begin{array}{l}a_{2} \\
e_{-} \\
a_{2} \ldots \ldots \\
a_{-} \ldots \ldots\end{array}$ & $\begin{array}{l}1 \\
2 \\
3 \\
4 \\
5\end{array}$ \\
\hline 10. 12511 & Methyl $\alpha$-D-lyxopyranoside_..... & 5 & $\mathrm{CA}, \mathrm{CE}^{\mathrm{e}}$. & $a, e^{\mathrm{e}}-$ & 13 & $\mathrm{CA}+\mathrm{CE}$; non- & $a+e ; a, e$, or $q$ & 6 \\
\hline 10. 12511 & Methyl $\beta$-D-lyxopyranoside & 4 & $\mathrm{CA}, \mathrm{CE}^{\mathrm{e}}$ & $e, a^{\mathrm{e}} \ldots$ & 13 & $\mathrm{CA}+\mathrm{CE}$; non- & $a+e ; a, e$ or $q$ & 7 \\
\hline 10. 22111 & 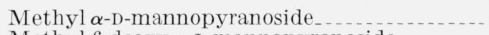 & 6 & $\mathrm{CA}_{-}$ & $a_{-.}$ & 13 & $\mathrm{CA}_{-}$ & & 8 \\
\hline 10. $22111(6) 80$ & Methyl 6-deoxy- $\alpha$-L-mannopyranoside $\ldots \ldots \ldots$ & 1 & $\mathrm{CA}_{-}$ & $a_{-.}$ & 13 & & & 9 \\
\hline 10. $22211(6) 80$ & Methyl 6-deoxy- $\beta$-L-mannopyranoside & 7 & (n) & (n)..... & .... & $\mathrm{CA}_{-}$ & & 10 \\
\hline $\begin{array}{l}\text { 10. } 42111 \\
10.2651199\end{array}$ & $\begin{array}{l}\text { Methyl D-glycero- } \alpha \text {-L-manno-heptopyranoside.. } \\
\text { Methyl } \alpha \text {-D-gulopyranoside, monohydrate... }\end{array}$ & $\begin{array}{l}8 \\
9\end{array}$ & $\mathrm{CA}, \mathrm{CE}$ & $a, e \ldots \ldots$ & 13 & $\mathrm{CA}+\mathrm{CE}$; non- & $a+e ; a, e$, or $q$ & $\begin{array}{l}11 \\
12\end{array}$ \\
\hline 10. $26 ? 119899$ & $\begin{array}{r}\text { Methyl } \alpha \text {-D-gulopyranoside } \cdot 1 / 2 \quad\left(\mathrm{CaCl}_{2} \cdot\right. \\
\left.3 \mathrm{H}_{2} \mathrm{O}\right) .\end{array}$ & 9 & & & & & & 13 \\
\hline 10. 26211 & Methyl $\boldsymbol{\beta}$-D-gulopyranoside & 9 & CA. & $e_{-.}$ & 15 & $\mathrm{CA}$ & $e_{-}$ & 14 \\
\hline 10. 36111 & Methyl D-glycero- $\alpha$-D-gulo-heptopyranoside & $\begin{array}{l}4 \\
4\end{array}$ & & $\cdots$ & ... & $\mathrm{CA}$ & & 15 \\
\hline 10. $36 ? 119899$ & $\begin{array}{l}\text { Methyl D-glycero- } \alpha \text {-D-gulo-heptopyranoside } \\
\mathrm{CaCl}_{2} \cdot \mathrm{H}_{2} \mathrm{O} \text {. }\end{array}$ & 4 & & & & & & 16 \\
\hline $\begin{array}{l}\text { 10. } 36211 \\
10.36 ? 119899\end{array}$ & $\begin{array}{l}\text { Methyl D-glycero- } \beta \text {-D-gulo-heptopyranoside } \\
\text { Methyl D-glycero- } \beta \text {-D-gulo-heptopyranoside }\end{array}$ & $\begin{array}{r}1,4 \\
4\end{array}$ & & & & $\mathrm{CA}_{-}$ & & $\begin{array}{l}17 \\
18\end{array}$ \\
\hline 10. 36213 & $\begin{array}{l}1 / 2 \mathrm{CaCl}_{2} \cdot \mathrm{H}_{2} \mathrm{O} \text {. } \\
\text { Cyclohexyl D-glycero- } \boldsymbol{\beta} \text {-D-gulo-heptopyranoside_ }\end{array}$ & 10 & & & & $\mathrm{CA}_{-}$ & $e_{-}$. & 19 \\
\hline 10. 13411 & Methyl $\alpha$-L-arabinopyranoside & 2 & CE_.. & $e_{-\ldots}$ & 16 & $\mathrm{CE}_{-}$ & & 20 \\
\hline 10. 13311 & Methyl $\beta$-L-arabinopyranoside & 2 & $\mathrm{CE}_{-.}$ & 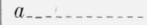 & 16 & $\mathrm{CE}_{-}$ & & 21 \\
\hline 10. 2311199 & Methyl $\alpha$-D-galactopyranoside, monohydrate.. & 1,11 & $\mathrm{CA}$ & $a_{-\ldots}+$ & 16 & $\mathrm{CA}$ & & 22 \\
\hline 10. 23211 & Methyl $\beta$-D-galactopyranoside & 1 & $\mathrm{CA} \ldots . .$. & $e_{\ldots} \ldots \ldots$ & 16 & $\mathrm{CA}_{-}$ & & 23 \\
\hline 10. 23111(6) 80 & Methyl 6-deoxy- $\alpha$-L-galactopyranoside $\ldots . . . . . .$. & 12 & 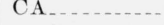 & $a_{-\ldots \ldots} \ldots \ldots$ & 16 & $\mathrm{CA}_{\ldots} \ldots . .$. & & 24 \\
\hline
\end{tabular}

a The third figure after the point was inserted after the present conclusions as to conformation had been reached.

b Named by the system of H. S. Isbell and R. S. Tipson, Science 130, 793 (1959); J. Research NBS 64A, 171 (1960). c Assignment made by Reeves [13 to 16] from consideration of instability factors. d After accepting several of Reeves' assignments (see text).

e Reeves later suggested [Ann. Rev. Biochem. 27, 15 (1958)] that the stable conformation may be a member of the boat-skew cycle. 
1. E. Fischer, Ber, deut. chem. Ges, 28, 1145 (1895).

2. C. S. Hudson, J. Am. Chem. Soc. 47, 265 (1925).

4. H. S. Isbell and H. L. Frush, J. Research NBS 24, 125 (1940) RP1274.

5. F. P Phelps and C S. Hudson, J Am. Chem 24, 125 (1940) RP1274

6. E. Fischer and L. Beensch, Ber, deut, chem. Ges, 29, 2927 (1896).

7. E. Fischer, M. Bergmann, and A. Rabe, Ber. deut. chem. Ges. 53, 2362 (1920)

8. R. M. Hann, A. T. Merrill, and C. S. Hudson, J. Am. Chem. Soc. 5̃, 2100 (1935).

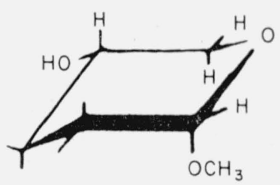

Q-D-C A

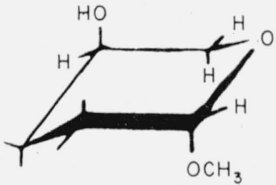

$\beta-L-C E$

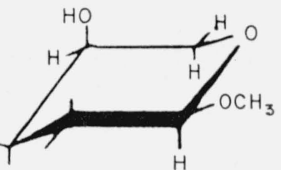

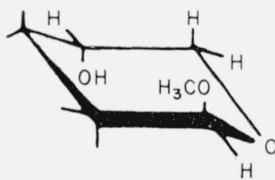

$\beta-D-C E$

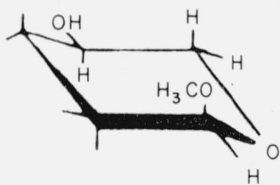

$a-L-C A$

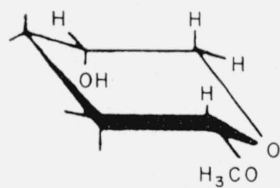

$a-D-C E$

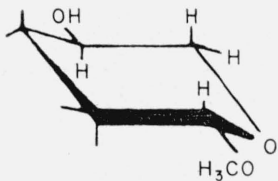

$\beta-L-C A$

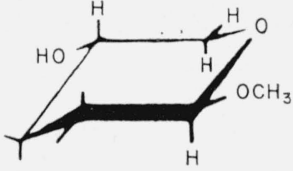

$\beta-D-C A$
9. H. S. Isbell, BS J. Research 8, 1 (1932) R P396.

10. E. Glaser and V Zuckermann, Z physiol. Chem 166, 103 (1927).

11. E Fischer and L Beensch, Ber deut chem. Ges, 27, 2478 (1894).

12. J. Minsaas, Rec tray chim 51, 475 (1932)

13. R. E Reeves, J Am. Chem. Soc. 72,1499 (1950).

14. R. E. Reeves, J. Am. Chem. Soc. 71, 215 (1949)

5. R. E. Reeves, Advances in Carbohydrate Chem. 6, 107 (1951)

16. R. E. Reeves, J. Am. Chem. Soc. 71, 1737 (1949).<smiles>[CH]1[CH-]C1</smiles>

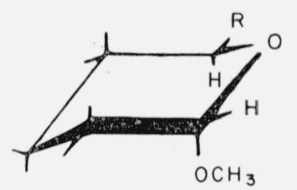

$\alpha-D-C A$

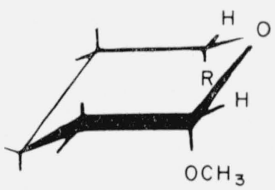

$\beta-L-C E$

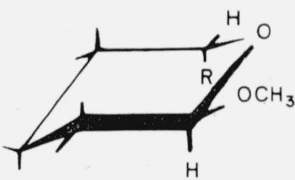

$a-L-C E$

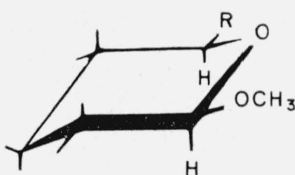

$\beta-D-C A$
$a-D-C E$

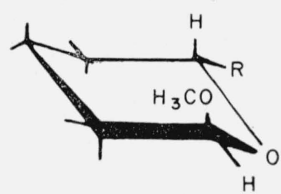

$\alpha-L-C A$

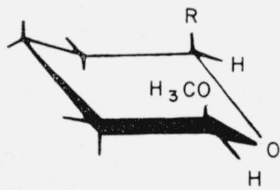

$\beta-D-C E$
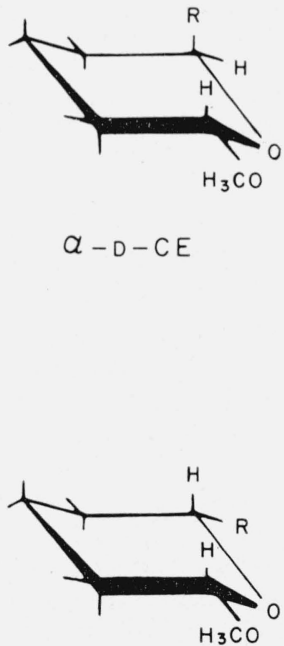

$\beta-L-C A$
Figure 1. The four general chair-forms of the anomeric methyl aldopentopyranosides, and the enantiomorph of each.

(The configurations at $\mathrm{C} 2$ and $\mathrm{C} 3$ are omitted.)

FIgure 2. The four general chair-forms of the anomeric methyl 6-deoxyaldohexopyranosides $\left(\mathrm{R}=\mathrm{CH}_{3}\right), \quad$ methyl aldohexopyranosides $\left(\mathrm{R}=\mathrm{CH}_{2} \mathrm{OH}\right)$, and methyl aldoheptopyranosides $\left(\mathrm{R}=\mathrm{CHOH}-\mathrm{CH}_{2} \mathrm{OH}\right)$, with the enantiomorph of each.

(The configurations at C2, C3, and $\mathrm{C} 4$ (and at C6, if asymmetric) are omitted.) 
For compounds $1,2,3,4,8,9,20,21,22,23$, and 24 (see table 1), the conformation predicted for each (by Reeves) as the most stable of all possibilities was found by him to be that actually adopted in cuprammonia; these glycosides comprise group A. Compounds 6, 7, and 12 form group B. For the anomers of methyl D-lyxopyranoside (compounds 6 and 7), Reeves at first predicted approximately equal stability for the CA and CE conformation of each, and his experimental results were in accord with the possibility of a mixture of the two conformations in cuprammonia; but he later suggested [9] that each might actually adopt one of the conformations in the boat-skew cycle. As regards compound 12 (methyl $\alpha$-D-gulopyranoside), Reeves predicted approximately equal stabilities for the $\mathrm{CA}$ and $\mathrm{CE}$ conformations, but his experimental results indicated that the CA conformation is adopted in cuprammonia.

In addition to the spectra of these 14 glycosides studied by Reeves, those of 10 aldopyranosides of hitherto undetermined conformation have been recorded and analyzed; conformations have now been assigned to 7 of these glycosides. As regards compound 14 (methyl $\beta$-D-gulopyranoside), Reeves predicted that the $\dot{C A}$ conformation would be the most stable of all conformations, but he did not examine the behavior of this glycoside in cuprammonia.

Barker and Shaw [10] have devised a way of predicting the stable chair-conformation of each pyranose, which involves the assumption "that the degree of distortion in a molecule is determined by the total amount of overlap of non-bonded atoms, overlap between each pair being calculated separately and added together." However, whereas a single, large overlap of $x$ units might prohibit the adoption of a certain ideal conformation, a number of small overlaps (whose sum is equal to, or greater than, $x$ units) might be accommodated by very slight departure of the conformation from ideality, so that, from the practical standpoint, the conformation in question is only slightly distorted. For this reason, the stable chair-conformations predicted by Reeves [6 to 8] have been used in the present study.

\section{Classification of the Glycosides into Configurationally Related Groups}

The 24 compounds were classified into three groups; the members of each group have like configurational features.

\subsection{Aldopyranosides of the xylo Configuration}

The members of this group of methyl aldopyranosides have the following general formulas (I) for the two chair-conformations.
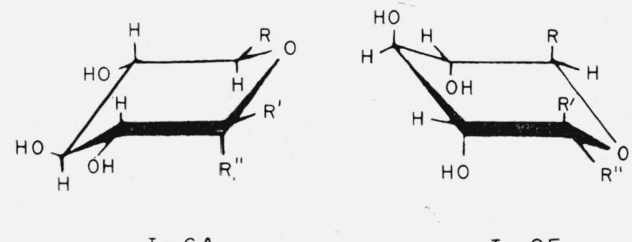

$$
I-C A
$$$$
I-C E
$$

Compounds 1 to 5 presumably have one $T$ the above general structures, with the following substituents.

1. Methyl $\alpha$-D-xylopyranoside, $\quad \mathrm{R}=\mathrm{H} ; \quad \mathrm{R}^{\prime}=\mathrm{H}$; and $\mathrm{R}^{\prime \prime}=\mathrm{OCH}_{3}$.

2. Methyl $\beta$-D-xylopyranoside, $\mathrm{R}=\mathrm{H} ; \mathrm{R}^{\prime}=\mathrm{OCH}_{3}$; and $\mathrm{R}^{\prime \prime}=\mathrm{H}$.

3. Methyl $\alpha$-D-glucopyranoside, $\mathrm{R}=\mathrm{CH}_{2} \mathrm{OH} ; \mathrm{R}^{\prime}=$ $\mathrm{H}$; and $\mathrm{R}^{\prime \prime}=\mathrm{OCH}_{3}$.

4. Methyl $\beta$-D-glucopyranoside, $\mathrm{R}=\mathrm{CH}_{2} \mathrm{OH} ; \mathrm{R}^{\prime}=$ $\mathrm{OCH}_{3}$; and $\mathrm{R}^{\prime \prime}=\mathrm{H}$.

5. Methyl D-glycero $\alpha$-L-gluco-heptopyranoside (originally called "methyl $\alpha$-D- $\beta$-galaheptopyranoside"),

$\mathrm{R}$ is $\mathrm{HCOH}$

$\mathrm{CH}_{2} \mathrm{OH} ; \mathrm{R}^{\prime}=\mathrm{H} ; \mathrm{R}^{\prime \prime}=\mathrm{OCH}_{3}$; and the molecule is the mirror image of that depicted.

The following names (which have no official status) may be applied to compounds 3 to 5 .

3. Methyl D-glycero- $a-\mathrm{D}-x y l o$-hexopyranoside.

4. Methyl D-glycero- $\beta$-D-xylo-hexopyranoside.

5. Methyl D-threo-a-L-xylo-heptopyranoside.

It should be noted that, in the CA conformation of compounds 2 and 4, all reference groups are equatorial; in the $\mathrm{CE}$ conformation of these compounds, all reference groups are axial.

\subsection{Aldopyranosides of the $1 y x o$ Configuration}

Three of the members of this group of configurationally related methyl aldopyranosides have the D-lyxo or D-manno configuration and the following general formulas (II) for the two chair-conformations.
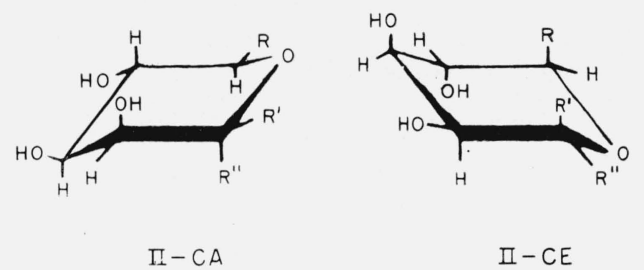

Compounds 6 to 8 , when in one of the chair conformations, have one of the above general structures, with the following substituents.

6. Methyl $\alpha$-D-lyxopyranoside, $\mathrm{R}=\mathrm{H} ; \mathrm{R}^{\prime}=\mathrm{H}$; and $\mathrm{R}^{\prime \prime}=\mathrm{OCH}_{3}$.

7. Methyl $\beta$-D-lyxopyranoside, $\mathrm{R}=\mathrm{H} ; \mathrm{R}^{\prime}=\mathrm{OCH}_{3}$; and $\mathrm{R}^{\prime \prime}=\mathrm{H}$.

8. Methyl $\alpha$-D-mannopyranoside, $\quad \mathrm{R}=\mathrm{CH}_{2} \mathrm{OH}$; $\mathrm{R}^{\prime}=\mathrm{H}$; and $\mathrm{R}^{\prime \prime}=\mathrm{OCH}_{3}$.

(Purely for comparative purposes, compound 8 can be regarded as methyl D-glycero- $\alpha$-D-lyxo-hexopyranoside; the name has no official status.)

Compounds 9 to 11 have the L-manno configuration; the following general formulas (III) depict the two chair-conformations, which are essentially the mirror images of formulas II. 
III $-C A$

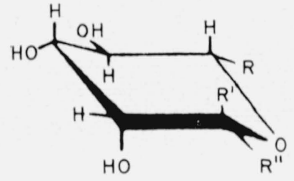

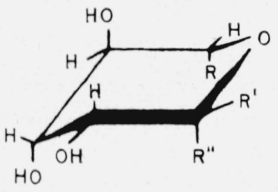

III $-C E$
When existing in one of the chair conformations, compounds 9 to 11 have one of the above general structures, with the following substituents.

9. Methyl 6-deoxy- $\alpha$-L-mannopyranoside (methyl $\alpha$-L-rhamnopyranoside), $\mathrm{R}=\mathrm{CH}_{3} ; \mathrm{R}^{\prime}=\mathrm{OCH}_{3}$; and $\mathrm{R}^{\prime \prime}=\mathrm{H}$.

10. Methyl 6-deoxy- $\beta$-L-mannopyranoside (methyl $\beta$-L-rhamnopyranoside), $\mathrm{R}=\mathrm{CH}_{3} ; \mathrm{R}^{\prime}=\mathrm{H}$; and $\mathrm{R}^{\prime \prime}=\mathrm{OCH}_{3}$.

11. Methyl D-glycero- $\alpha$-L-manno-heptopyranoside (originally called "methyl $\alpha$-D- $\alpha$-galaheptopyranoside"),

$\mathrm{R}$ is $\mathrm{HCOH}$

$$
\mathrm{CH}_{2} \mathrm{OH} ; \mathrm{R}^{\prime}=\mathrm{OCH}_{3} \text {; and } \mathrm{R}^{\prime \prime}=\mathrm{H} \text {. }
$$

To emphasize the configurational relationships, the following unofficial names may be temporarily applied:

9. Methyl 6-deoxy-L-glycero- $a$-L-lyxo-hexopyranoside.

10. Methyl 6-deoxy-L-glycero- $\beta$-L-lyxo-hexopyranoside.

11. Methyl D-threo-a-L-lyxo-heptopyranoside.

Compounds 12 to 19 have the D-gulo configuration; the following general formulas (IV) depict their chair conformations, which are closely related to formulas III.

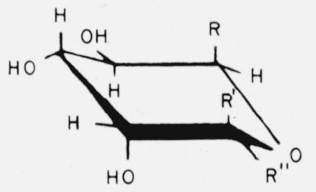

$I V-C E$

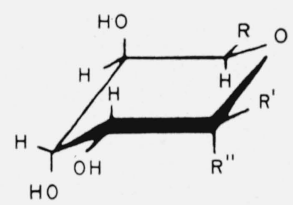

IV-CA
12. Methyl $\alpha$-D-gulopyranoside monohydrate, $\mathrm{R}=\mathrm{CH}_{2} \mathrm{OH} ; \mathrm{R}^{\prime}=\mathrm{H}$; and $\mathrm{R}^{\prime \prime}=\mathrm{OCH}_{3}$.

14. Methyl $\beta$-D-gulopyranoside, $\quad \mathrm{R}=\mathrm{CH}_{2} \mathrm{OH}$; $\mathrm{R}^{\prime}=\mathrm{OCH}_{3}$; and $\mathrm{R}^{\prime \prime}=\mathrm{H}$.

15. Methyl D-glycero- $\alpha$-D-gulo-heptopyranoside (originally called "methyl $\alpha$-D- $\alpha$-glucoheptopyranoside"),

$\mathrm{R}$ is $\mathrm{HCOH}$

$\mathrm{CH}_{2} \mathrm{OH} ; \mathrm{R}^{\prime}=\mathrm{H}$; and $\mathrm{R}^{\prime \prime}=\mathrm{OCH}_{3}$.

17. Methyl D-glycero- $\beta$-D-gulo-heptopyranoside (originally called "methyl $\beta$-D- $\alpha$-glucoheptopyranoside"),

\section{$\mathrm{R}$ is $\mathrm{HCOH}$}

$\mathrm{CH}_{2} \mathrm{OH} ; \mathrm{R}^{\prime}=\mathrm{OCH}_{3}$; and $\mathrm{R}^{\prime \prime}=\mathrm{H}$.
19. Cyclohexyl D-glycero- $\beta$-D-gulo-heptopyranoside,

$\mathrm{R}$ is $\mathrm{HCOH}$

$$
\mathrm{CH}_{2} \mathrm{OH} ; \mathrm{R}^{\prime}=\mathrm{OC}_{6} \mathrm{H}_{11} ; \mathrm{R}^{\prime \prime}=\mathrm{H} .
$$

For comparison with compounds 6 to 8 , and 9 to 11, the following unofficial names may be temporarily applied:

12. Methyl D-glycero- $\beta$-L-lyxo-hexopyranoside, monohydrate.

14. Methyl D-glycero- $a$-L-lyxo-hexopyranoside.

15. Methyl D-erythro- $\beta$-L-lyxo-heptopyranoside.

17. Methyl D-erythro- $\alpha-\mathrm{L}-l y x o$-heptopyranoside.

19. Cyclohexyl D-erythro-a-L-lyxo-heptopyranoside.

Because the $\alpha-\beta$ names are relative, not absolute, it will be noted that, whenever $C_{4}$ and $C 5$ of a sugar have opposite configurations, the CA conformation for the sugar when named as a substituted L-aldopentopyranose is the CE conformation when it is named as a D-aldohexopyranose.

\subsection{Aldopyranosides of the arabino Configuration}

This group of configurationally related glycosides consists of the methyl L-arabinopyranosides, the methyl D-galactopyranosides, and methyl 6-deoxy$\alpha$-L-galactopyranoside. The relationship of the Larabinopyranosides to the $\mathrm{D}$-galactopyranosides is the same as that of the L-lyxopyranosides to the D-gulopyranosides.

Compounds 20 to 23 may have one of the following structures (V and VI), with the indicated substituents.

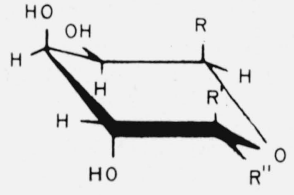

E

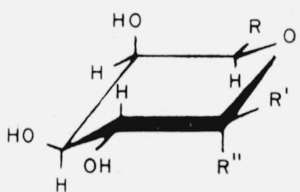

VI
20. Methyl $\alpha$-L-arabinopyranoside, $\quad \mathrm{R}=\mathrm{H} ; \quad \mathrm{R}^{\prime}$ $=\mathrm{OCH}_{3}$; and $\mathrm{R}^{\prime \prime}=\mathrm{H}$.

21. Methyl $\beta$-L-arabinopyranoside, $\mathrm{R}=\mathrm{H} ; \mathrm{R}^{\prime}=\mathrm{H}$; and $\mathrm{R}^{\prime \prime}=\mathrm{OCH}_{3}$.

22. Methyl $\alpha$-D-galactopyranoside, monohydrate, $\mathrm{R}=\mathrm{CH}_{2} \mathrm{OH} ; \mathrm{R}^{\prime}=\mathrm{H} ;$ and $\mathrm{R}^{\prime \prime}=\mathrm{OCH}_{3}$.

23. Methyl $\beta$-D-galactopyranoside, $\mathrm{R}=\mathrm{CH}_{2} \mathrm{OH} ; \mathrm{R}^{\prime}$ $=\mathrm{OCH}_{3}$; and $\mathrm{R}^{\prime \prime}=\mathrm{H}$.

For purposes of comparison, compounds 22 and 23 may be temporarily given the following unofficial names:

22. Methyl D-glycero- $\beta$-L-arabino-hexopyranoside, monohydrate.

23. Methyl D-glycero- $a$-L-arabino-hexopyranoside.

Named in this way, the CE conformation is the same as the conformation that is called CA when they are named as Daldohexopyranosides.

For compounds 20 and 21 , formula $\mathrm{V}$ represents the CA conformation and formula VI represents the CE conformation. For compounds 22 and 23, named as D-aldohexopyranosides, formula $\mathrm{V}$ represents the $\mathrm{CE}$ conformation and formula VI represents the CA conformation.

The possible chair conformations of compound 24 are the mirror images of those depicted above, with the following substituents. 
24. Methyl 6-deoxy- $\alpha$-L-galactopyranoside (methyl $\alpha$-L-fucopyranoside), $\mathrm{R}=\mathrm{CH}_{3} ; \mathrm{R}^{\prime}=\mathrm{OCH}_{3}$; and $\mathrm{R}^{\prime \prime}=\mathrm{H}$.

The compound may, for purposes of comparison, be unofficially called methyl 6-deoxy-L-glycero- $\beta$-D-arabino-hexopyranoside. Named in this way, the conformation $V$ having an axial $(\beta-\mathrm{D})$ anomeric group is classified as $\mathrm{CE}$; named as an $a-\mathrm{L}$-hexopyranoside, it is classified as CA.

\section{Discussion of the Spectra}

In the present investigation, the positions of the various absorption bands for each of 24 aldopyranosides have been determined. For reasons discussed later, the relative intensities of absorption were not examined in detail.

The predicted stable conformations of 14 of these glycosides [6 to 8] are listed in table 1 ; eleven of these were accepted, but three (for compounds 6,7 , and 12) seemed open to question. In order that the conformation of these three and of each of the 10 remaining glycosides might be determined, the problem then resolved itself into a search of the infrared absorption spectra of the 11 "known" glycosides for potentially distinctive bands, followed by attempts to correlate the positions of these bands with such conformational and structural features as axial or equatorial disposition of (a) the glycosidic aroup at C1 or (b) the reference group (if any) at C5. The resultant findings were then applied in a study of the spectra of the glycosides of unknown conformation, in order to assign a conformation to each (in the solid state).

The spectra were examined in the groups outlined in table 2. However, before proceeding to a detailed analysis (see sections 5.3 and 5.4), some preliminary studies were made (in order to determine which methods of approach were likely to be the most fruitful).

\subsection{Effect of Calcium Chloride of Crystallization}

Glycosides 12, 15, and 17 lacked the following bands shown by their compounds with calcium chloride (group 1 of table 2): 3226 to $3215 \mathrm{~cm}^{-1}$;

TABLE 2. Structural groups studied

\begin{tabular}{|c|c|c|}
\hline Group & Structural feature & $\begin{array}{l}\text { Compounds (serial } \\
\text { numbers) in group }\end{array}$ \\
\hline 1 & Calcium chloride (of crystallization) & $13,16,18$ \\
\hline 2 & arabino; galacto configuration ........ & 20,$21 ; 22$ to 24 \\
\hline 3 & lyxo; manno; gulo configuration... & $\begin{array}{l}6,7: 8 \text { to } 11 ; 12,14,15,17,19 \\
{[13,16,18] .}\end{array}$ \\
\hline 4 & xylo; gluco configuration. - & 1,$2 ; 3$ to 5 \\
\hline 5 & Pentopyranoside $\ldots \ldots$ & $1,2,6,7,20,21$. \\
\hline 6 & 5-C-Methyl (6-deoxy) group & $9,10,24$ \\
\hline 7 & $\begin{array}{l}\text { Hexopyranoside; } 5 \text {-C-(hydroxymethyl) } \\
\text { group. }\end{array}$ & $3,4,8,12,14,22,23[13]$. \\
\hline 8 & $\begin{array}{l}\text { Heptopyranoside; } 5 \text { - } C \text {-(1,2-dihydroxyeth- } \\
\text { yl) group. }\end{array}$ & $5,11,15,17,19[16,18]$ \\
\hline 9 & Hydrate & $12,13,16,18,22$ \\
\hline 10 & Cyclohexyl group, glycosidic & 19. \\
\hline 11 & Methoxyl group, glycosidic & $\begin{array}{l}1 \text { to } 12,14,15,17,20 \text { to } 24 \\
{[13,16,18] \text {. }}\end{array}$ \\
\hline 12 & Hydroxyl group, primary & 3 to $5,8,11,12,14,15,17,19$, \\
\hline 13 & Hydroxyl group, secondary .......... & 1 to $12,14,15,17,19$ to 24 \\
\hline 14 & Pyranoid ring & f $[13,16,18]$ \\
\hline
\end{tabular}

$1745 \mathrm{~cm}^{-1} ; 1366$ to $1364 \mathrm{~cm}^{-1} ; 1148$ to $1130 \mathrm{~cm}^{-1}$; 1117 to $1114 \mathrm{~cm}^{-1} ; 1049$ to $1044 \mathrm{~cm}^{-1} ; 772$ to 771 $\mathrm{cm}^{-1}$; and 655 to $643 \mathrm{~cm}^{-1}$. Because co-crystallization with calcium chloride alters the spectrum, either by removal or displacement of certain bands, only the spectra of the 21 compounds lacking calcium chloride were intercompared with respect to the other structural, configurational, and conformational features (groups 2 to 14 of table 2 ).

\subsection{Preliminary Evaluation of Configurational and Constitutional Effects}

Kuhn [11] recorded the spectra (in the range of 1250 to $667 \mathrm{~cm}^{-1}$ ) for compounds $2,3,4,8,22$, and 23. He noted that "the difference between the anomeric forms shows up very nicely." In table 1 are listed eight anomeric pairs, namely 1,$2 ; 3,4$; 6,$7 ; 9,10 ; 12,14 ; 15,17 ; 20,21 ;$ and 22,23 . A cursory inspection of their spectra revealed that Kuhn's remark applies to all of these pairs.

When the two spectra for an anomeric pair are compared, it is seen that (a) they have certain bands "in common" and (b) each shows certain bands that are "absent" from the other. With the information at hand, it was not known whether the absence of a band in one spectrum (and its presence in the other spectrum) is real or is actually due to a shift to some other position. If the "absent" band has actually been shifted to a position matching a band in the other spectrum, it will temporarily be regarded as being a band " common to the two spectra." On the other hand, if a band is shifted to a position not matched in the other spectrum, it will be observed as being "present" as a distinguishing band. Hence, some bands that may actually be ascribable to different features may be paired for the two spectra, and some bands (in the two spectra) that are actually ascribable to the same feature may appear to differentiate between the two.

Nevertheless, if the bands "differentiating" the two members of one anomeric pair are found to bear some relationship to the bands differentiating the two members of a different anomeric pair, it is reasonable to ascribe this to some influence that is operative in both instances.

For molecules as complex as those of the aldopyranosides, many of the observed bands cannot yet be assigned to particular vibrational modes. Assignments for some of the bands are given in section 5.5. However, we are not here concerned with (a) which bands, arising from vibrations localized in a functional group, are relatively independent of the remainder of the molecule, or (b) which bands involve other parts of the molecule (and are, therefore, sometimes perturbed in unexpected ways when the molecule is altered). Instead, in the present treatment, a band in two different spectra is regarded as "a band common to the two spectra" if it occupies approximately the same position in the spectrum, regardless of (a) whether it is actually contributed by vibrations which are of the same nature in the two molecules, or (b) the relative intensity in the two spectra. In our analysis, all of these bands are given equal weight.

Before proceeding to a detailed study, an examination of effects possibly attributable to configuration and constitution was undertaken. Simultane- 
ously, an attempt was made to ascertain whether any of the differentiating bands could be regarded as being indicative of the axial or equatorial disposition of the anomeric group. It was realized that, if the anomeric group of an $\alpha$ anomer is axial, the conclusion that the anomeric group of its $\beta$ anomer is equatorial might not necessarily follow-the disposition might, for example, be quasi; and vice versa. Therefore, to avoid unconscious bias that might arise from the use of the customary $\alpha$ or $\beta$ names, only the assigned serial numbers of the above eight pairs of glycosides were used when making intercomparisons of their spectral characteristics.

Those of our compounds whose stable conformations had been assigned by Reeves [6 to 8] were all methyl glycosides. Consequently, from the total of 24 aldopyranosides listed in table 1, the cyclohexyl glycoside (compound 19) was excluded in this part of the study. Moreover, in view of the effects noted in section 5.1, the three compounds containing calcium chloride of crystallization (group 1 of table 2) were also excluded.

The generalized similarities and differences in the methyl aldopyranosides studied may be summarized as follows.

\begin{tabular}{|c|c|c|}
\hline C1 & $\mathrm{C} 2 \quad \mathrm{C} 3 \quad \mathrm{C} 4$ & C5 \\
\hline $\begin{array}{l}\text { Disposition } \\
\text { of } \mathrm{OCH}_{3}\end{array}$ & $\begin{array}{c}\text { Group- } \\
\text { configuration }\end{array}$ & Substituent \\
\hline $\begin{array}{ll} & a \\
\text { or } & e \\
\text { or } & q\end{array}$ & $\begin{aligned} & x y l o \\
& \text { or lyxo } \\
& \text { or arabino }\end{aligned}$ & $\begin{array}{ll} & \mathrm{H} \\
\text { or } & \mathrm{CH}_{3} \\
\text { or } & \mathrm{CH}_{2} \mathrm{OH} \\
\text { or } & \mathrm{CHOH}-\mathrm{CH}_{2} \mathrm{OH}\end{array}$ \\
\hline
\end{tabular}

No glycoside having the ribo group-configuration is included in this investigation.

\section{a. Effect of Change of Group-Configuration on Positions of Bands Common to Spectra (for Each Group-Configuration)}

By group-configuration is meant the configuration of the glycoside, regardless of its anomeric form (see groups 2,3 , and 4 of table 2 ). In this analysis, the infrared spectra were examined for eight anomeric pairs (see above). The bands common to any one pair of anomers were systematically compared with the bands common to each and every other pair of configurationally related anomers. The three pairs of methyl pentopyranosides available for study were not configurationally related; each pair of pentopyranosides was compared with the related 5-C-substituted derivatives. For any one anomeric pair of pentopyranosides, these comprised anomeric pairs (and a few single anomers) of one or more of the following derivatives: 5 - $C$-methyl, or methyl 6-deoxyaldohexopyranosides; 5- $C$-(hydroxymethyl), or methyl aldohexopyranosides; and $5-C$ (1,2-dihydroxyethyl), or methyl aldoheptopyranosides. Three such configurational groups were available for study, namely, groups 2, 3, and 4 of table 2. Essentially the same kind of procedure was adopted in examining each group, and so a description of the method used will be exemplified by discussion of its application to group 2 .

For group 2, the bands differentiating the $\alpha$ from the $\beta$ anomer of methyl L-arabinopyranoside were tabulated and set aside for later evaluation (see section 5.3), and those bands common to the two anomers were tabulated. Similarly, the bands differentiating the $\alpha$ from the $\beta$ anomer of methyl D-galactopyranoside were tabulated and set aside (see section 5.4), and the bands common to these two anomers were tabulated. It was then observed that introduction of the 5-C-(hydroxymethyl) group in the pentopyranoside, to afford the hexopyranoside, had resulted in the display of a different spectrum of bands. (The fact that the group-configuration was enantiomorphic may be ignored in this connection.) This observation is further developed in section 5.2.c.

In view of this effect of substitution at C5 of the pentopyranoside, a further winnowing of bands was undertaken. Those bands differentiating the pentopyranosides from the hexopyranosides were set aside for later consideration (see sections 5.3 and 5.4), and the bands common to the two pentopyranosides and the two hexopyranosides were tabulated. Finally, such of these bands as were also shown by the sole 6-deoxyaldohexopyranoside in this group (namely, compound 24) were selected and tabulated. This afforded a table recording the bands shown by all the glycosides (in this study) that have the arabino or galacto configurations.

In a similar manner, a table was compiled of the bands shown by all the members of group 3 (of table 2), and another table of the bands shown by all the members of group 4. These tables recorded, for each group-configuration, the bands that are shown regardless of anomeric disposition or of substitution (or nonsubstitution) at C5.

Inspection of these three tables revealed that a change in group-configuration results in changes in the positions of a number of bands, common to one configurational group, relative to those common to another configurational group. As a corollary, a set of bands shown by one group-configuration may tentatively be regarded as characteristic of that group-configuration.

Incidentally, on intercomparing the three groups, jt was noted that the 21 glycosides comprising groups 2,3 , and 4 have, after application of the winnowing deseribed, only four bands in common, namely, those at 2882 to $2841 \mathrm{~cm}^{-1}$, 1368 to $1330 \mathrm{~cm}^{-1}, 1153$ to $1111 \mathrm{~cm}^{-1}$, and 1109 to 1087 $\mathrm{cm}^{-1}$ (see sec. 5.5). These bands are displayed regardless of the anomeric disposition or of substitution (or nonsubstitution) at C5.

\section{b. Effect of Change of Group-Configuration on Positions of Bands Which Diff эrentiate Two Anomers}

The bands that differentiate the two members of each anomeric pair were tabulated; they are listed and discussed in sections 5.3 and 5.4. Study of these tables revealed that, for the aldopentopyran- 
osides, change from the xylo to the arabino configuration leaves the majority of the anomer-differentiating bands substantially unchanged in position (see sec. 5.3). For the 5- $\dot{C}$-substituted aldopentopyranosides, change from one configuration to another (e.g., of the aldohexopyranosides) causes changes in the positions of bands that differentiate anomers.

\section{c. Effect (on the Spectra) of Various Substitutions at Carbon Atom 5 of Aldopentopyranosides}

In this analysis, bands shown by an anomeric pair of aldopentopyranosides (group 5 of table 2 ) were first compared with those shown by 5 - $C$-substituted derivatives (groups 6,7 , and 8). It was found that substitution at $\mathrm{C} 5$ of the aldopentopyranosides caused shifts in the anomer-differentiating bands.

However, intercomparison of the spectra of the 5 - $C$-substituted derivatives revealed that, for any one group-configuration, change in the substituent from the methyl to the hydroxymethyl or to the 1,2 dihydroxyethyl group did not cause profound shifts of anomer-differentiating bands.

To summarize the observations in sections $5.2 \mathrm{a}, \mathrm{b}$, and $c$, it is seen that, for the diagnostic purposes under consideration, the spectra of the 5 - $C$-substituted aldopentopyranosides of any one groupconfiguration may be intercompared, but they should not be intercompared with the spectra of their isomers having a different group-configuration, nor with those of the related aldopentopyranosides. On the other hand, intercomparison of the spectra of members of groups 6,7 , and 8 that have the same configuration might afford fruitful results.

\subsection{Absorption Bands Possibly Indicative of the Axial or Equatorial Anomeric Group of the Methyl Aldopentopyranosides}

As the starting point in this series of analyses of spectra, we selected methyl $\beta$-D-xylopyranoside (compound 2) because, if this compound adopts a chair conformation, the reference groups will either be all axial (CE) or all equatorial (CA). Its spectrum was compared with that of its $\alpha$ anomer (compound 1 ), in order to determine the effect (on the spectrum) of changing the anomeric group from equatorial to axial, or vice versa. A similar comparison was now made for the anomers of methyl L-arabinopyranoside (compounds 20 and 21).

Bands that are essentially the same for both anomers of (a) the methyl D-xylopyranosides or of (b) the methyl L-arabinopyranosides, or of (c) both configurational groups, are given ${ }^{4}$ in table 3 . It seemed reasonable to assume tentatively that bands shown by all of these glycosides might be independent oi total configuration, whereas those shown by one pair of anomers having the same group-configuration might be a reflection, via shifting of bands, of an effect of the total configuration of that pair.

${ }^{4}$ For comparison, bands (in about the same positions) shown by either or both anomers of methyl D-lyxopyranoside are also given in table 3.
TABLE 3. Bands $\left(\mathrm{cm}^{-1}\right)$ shown by both anomers of methyl D-xylopyranoside or by both anomers of methyl L-arabinopyranoside (or by all four compounds); and positionally corresponding bands of the methyl D-lyxopyranosides

\begin{tabular}{|c|c|c|c|c|c|}
\hline \multicolumn{2}{|c|}{ Methyl D-xylopyranosides } & \multicolumn{2}{|c|}{$\begin{array}{l}\text { Methyl L-arabino- } \\
\text { pyranosides }\end{array}$} & \multicolumn{2}{|c|}{$\begin{array}{l}\text { Methyl D-lyxo- } \\
\text { pyranosides }\end{array}$} \\
\hline 2 & 1 & 20 & 21 & 6 & 7 \\
\hline
\end{tabular}

Possibly nonconfigurational bands

\begin{tabular}{|c|c|c|c|c|c|}
\hline 2950 & 2924 & 2941 & 2950 & 2924 & 2924 \\
\hline 2849 & 2841 & 2857 & 2841 & 2841 & 2849 \\
\hline 1473 & 1466 & 1464 & 1466 & 1466 & 1471 \\
\hline $1451 \ldots$ & 1449 & 1456 & 1453 & 1456 & 1451 \\
\hline 1410 & 1418 & 1429 & 1422 & 1414 & 1429 \\
\hline 1376 & 1379 & 1379 & 1376 & 1377 & 1383 \\
\hline 1368 & 1362 & 1353 & 1362 & 1359,1355 & 1353 \\
\hline 1277 & 1269 & 1266 & 1269 & 1274 & 1280 \\
\hline 1242 & 1247 & 1258 & 1245 & 1245,1241 & 1235 \\
\hline $1192 \ldots$ & 1195 & 1205 & 1192 & 1199 & 1211 \\
\hline $1161 \ldots$ & 1147 & 1166 & 1145 & 1151 & 1160 \\
\hline 1119 & 1119 & 1116 & 1119 & 1106,1103 & 1106 \\
\hline $1091 \ldots$ & 1091 & 1099 & 1099 & 1086 & 1089 \\
\hline $898 \mathrm{a}_{\ldots} \ldots$ & a 897 & 877 & 883 & 880 & 885 \\
\hline $404\left(399{ }^{\prime}\right)$ & 400 & $(396 ?)$ & 391 & 401 & (398?) 389 \\
\hline
\end{tabular}

Bands possibly affected by configuration and conformation

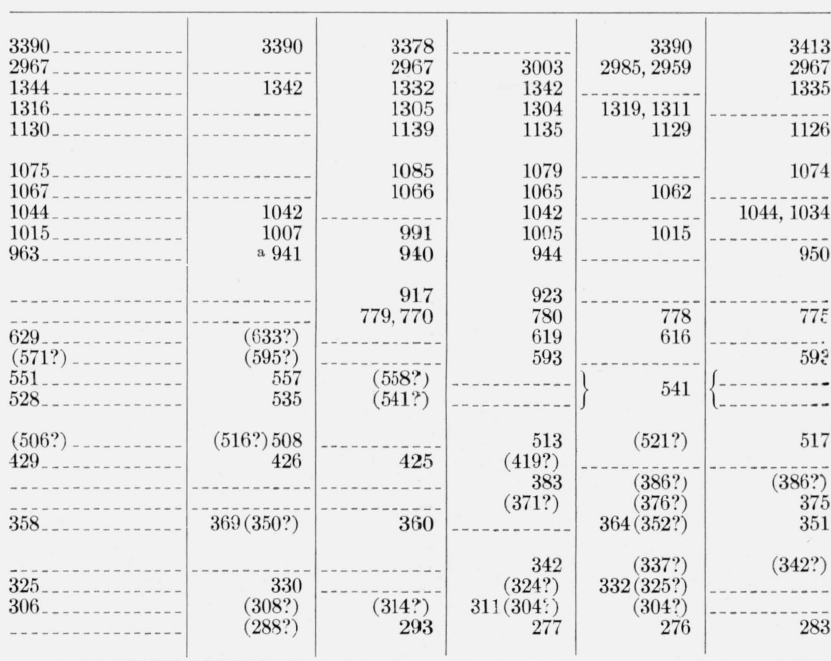

a These bands were mentioned by S. A. Barker, E. J. Bourne, R. Stephens, and D. H. Whiffen, J. Chem. Soc. 1954, 3468 .

In table 4 are given the bands shown by one anomer (but not the other) of the methyl D-xylopyranosides and the methyl L-arabinopyranosides. If these "anomer-differentiating" bands have any relationship to the axial or equatorial disposition of the respective glycosidic methoxyl group, the results in table 4 indicate that (a) compounds 2 and 20 have the same anomeric disposition, and (b) compounds 1 and 21 have the same anomeric disposition; that is, in both instances, both are equatorial or both are axial. If the conformation predicted by Reeves $[7,8]$ as being the most stable for any one of these four compounds is accepted for the crystalline state, the conformations of the other three may be deduced from the results in table 4 . For example, if the anomeric group of methyl $\beta$-D-xylopyranoside is equatorial and that of its $\alpha$ anomer is axial, the results indicate that the anomeric group of methyl 
TABLE 4. Bands $\left(\mathrm{cm}^{-1}\right)$ shown by only one anomer of the methyl xylopyranosides and methyl arabinopyranosides, compared with bands for both anomers of methyl lyxopyranoside

\begin{tabular}{|c|c|c|c|c|c|}
\hline \multicolumn{2}{|c|}{ Methyl D-xylopyranosides } & \multicolumn{2}{|c|}{$\begin{array}{l}\text { Methyl L-arabino- } \\
\text { pyranosides }\end{array}$} & \multicolumn{2}{|c|}{$\begin{array}{l}\text { Methyl D-lyxo- } \\
\text { pyranosides }\end{array}$} \\
\hline 2 & 1 & 20 & 21 & 6 & 7 \\
\hline \multicolumn{6}{|l|}{3448} \\
\hline 3012 & & & & 3209 & \\
\hline $\begin{array}{l}2874-- \\
1433-\end{array}$ & & - & & $\begin{array}{l}2882 \\
1445\end{array}$ & \\
\hline 1385 & & 1395 & & 1445 & 1408,1383 \\
\hline $1295 \ldots$ & & 1295 & & 1274 & 1280 \\
\hline $1218 \ldots$ & & $\begin{array}{l}1227 \\
1058\end{array}$ & & 1062 & \\
\hline $\begin{array}{l}976 \ldots \\
645\end{array}$ & & 973 & & 973 & 975 \\
\hline \multirow{2}{*}{\multicolumn{6}{|c|}{$\begin{array}{l}(496 ?) \\
473 \\
\end{array}$}} \\
\hline & & & & & 463 \\
\hline & 3333 & & 3322 & & 3367 \\
\hline & & & $\begin{array}{l}3236 \\
2801\end{array}$ & 3226 & \\
\hline & 2710 & $\cdots$ & 2695 & 2717,2667 & 2703 \\
\hline & a741 & & $\begin{array}{r}845,838 \\
744\end{array}$ & $\begin{array}{l}848 \\
744\end{array}$ & 865 \\
\hline & & & 713,699 & 685 & 697 \\
\hline & 437 & & $\begin{array}{l}678 \\
433\end{array}$ & 664 & \\
\hline & & & & & \\
\hline
\end{tabular}

a See footnote to table 3

$\alpha$-L-arabinopyranoside is equatorial and that that of its $\beta$ anomer is axial. These conclusions are in complete agreement with Reeves' assignments (see table 1 ). It may be noted that the sole difference between methyl $\beta$-D-xylopyranoside-CA and methyl $\alpha$-L-arabinopyranoside-CE lies in the configuration of carbon atom 4 .

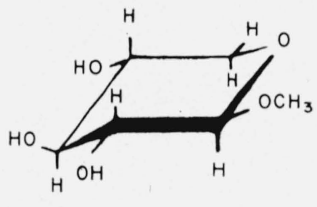

$2-C A$

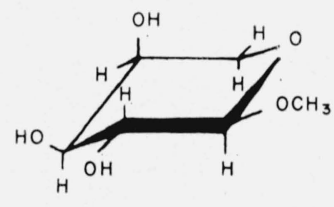

$20-C E$
The corresponding bands of the methyl D-lyxopyranosides are also given in table 4 . The results suggest that, for each anomer thereof, either (a) a conformation having a quasi or a different kind of axial or equatorial anomeric group is adopted in the crystalline state, or (b) a mixture of the CA and CE conformations crystallized together. These conclusions also agree with Reeves' predictions (see table 1).

It should be noted that, in this series of intercomparisons, there was no possibility of band displacements attributable to the effect of a substituent at carbon atom 5 . However, displacements (to lower or higher wavenumbers) caused by the differences in configuration might have been either (a) retained or (b) partially or wholly eliminated by the arbitrary procedure of temporarily ignoring bands that are essentially the same for both anomers of methyl D-xylopyranoside or of methyl L-arabinopyranoside, or both.

\subsection{Analysis of the Spectra of Groups of Con- figurationally Related Aldopyranosides, Excluding the Aldopentopyranosides}

In this analysis, two potential sources of misinterpretation were avoided. Firstly, intercomparisons were not made between the aldopentopyranosides and (a) the 6-deoxyaldohexopyranosides, (b) the aldohexopyranosides, and (c) the aldoheptopyranosides, because of the band displacements caused by the various substituents at $\mathrm{C} 5$ of the aldopentopyranosides. On the other hand, it was assumed that change from a methyl substituent at C5 to either a hydroxymethyl or a 1,2-dihydroxyethyl substituent would occasion no change in conformation; shifts of bands have already been discussed (see sec. 5.2). Secondly, intercomparisons were confined to intra-group study of groups of glycosides having the same configuration for each member of one group. In this way, band shifts attributable to change of configuration did not come into consideration.

First of all, as a check on the significance of the results accruing from our method of comparing absorption bands, the spectra of the anomers of methyl D-galactopyranoside (compounds $22 \uparrow$ and 23 ) were compared with that of methyl 6-deoxy- $\alpha-\mathrm{L}-$ galactopyranoside (compound 24). The results. are given in table 5; column $\mathrm{C}$ gives bands shown by the $\beta$ anomer but not by the two $\alpha$ anomers, and column $\mathrm{D}$ records bands shown by the two $\alpha$ anomers. but not by the $\beta$ anomer. It may be concluded that, assuming the validity of the correlations, if the anomeric group of the $\alpha$ anomers is axial, that of the $\beta$ anomer is equatorial, or vice versa. If the conformation predicted by Reeves [7] as being the most

TABLE 5. Comparison a of absorption bands $\left(\mathrm{cm}^{-1}\right)$ shown by the methyl D-galactopyranosides (22 and 23) and by methyl 6 -deoxy- $\alpha$-L-galactopyranoside (24)

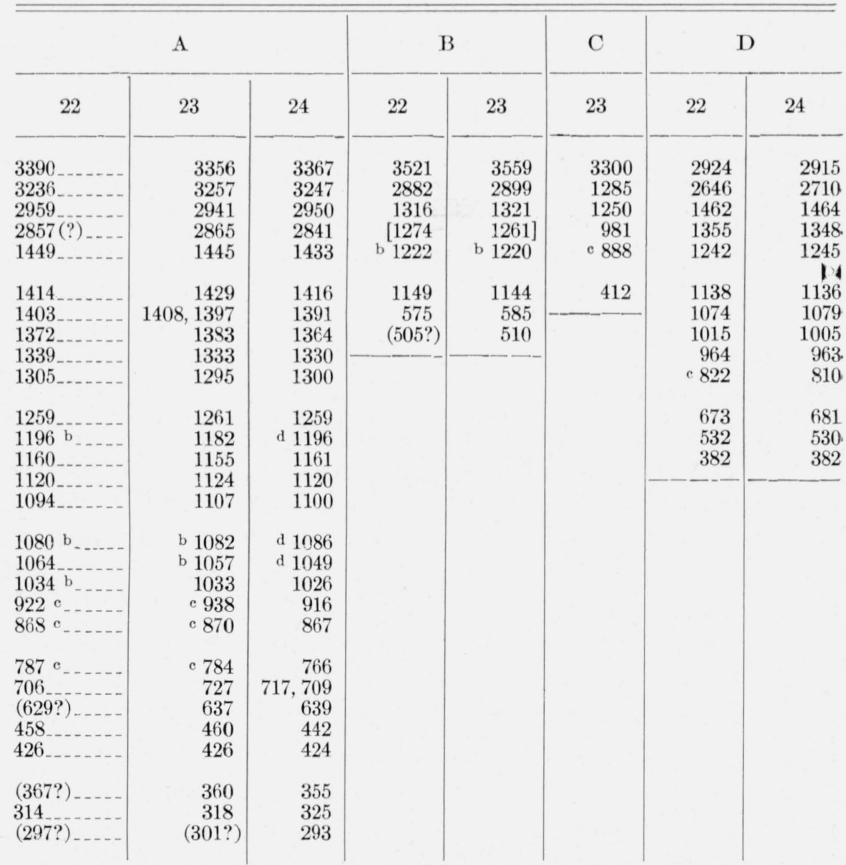

a Key: A. Bands shown by both anomers of methyl D-galactopyranoside and by methyl 6-deoxy- $\alpha$-L-galartopyranoside. B. Bands shown by both anomers of methyl D-galactopyranoside, but not by compound 24. C. Bands shown by methyl $\beta$-D-galactopyranoside, but not by methyl $\alpha$-D-galactopyranoside or methyl 6-deoxy- $\alpha$-L-galactopyranoside. D. Bands shown by methyl $\alpha$-Dgalactopyranoside and by methyl 6-deoxy- $\alpha$-L-galactopyranoside, but not by $\alpha$-Dpound 23 b These bands were mentioned by R L Whistler not by compound 23. b These bands were mentioned by R. L. Whistler and L. R. House, Anal. Chem. 25, $1463(1953)$. c See footnote $a$ to table 3 . d These
bands were mentioned (see footnote $b$ ) for the $\alpha$-D form. 
stable for any one of these three compounds is accepted for the crystalline state, the deduced conformations for the other two are in complete agreement with Reeves' assignments for them (see table 1).

The anomeric disposition of methyl $\alpha$-D-gulopyranoside (compound 12) was now studied. Its spectrum was compared with those of methyl $\alpha$-D-mannopyranoside (compound 8 ) and methyl $\dot{\beta}$-D-gulopyranoside (compound 14). The results are given in table 6 ; column $\mathrm{C}$ records bands shown by compound 12 that are absent from the spectra of compounds 8 and 14; column D gives the bands common to compounds 8 and 12 ; and column $\mathrm{E}$ gives the bands common to compounds 8 and 14 , not shown by compound 12. Thus, the spectrum of compound 12 has some resemblances to that of compound 8 and some to that of compound 14 , but it also differs from both. Now; for the most stable conformation of compound 8, Reeves [8] predicted an axial anomeric group ; for compound 14, he predicted [12] an equatorial anomeric group. The results in table 6 suggest that, if these assignments are accepted, compound 12 in the crystalline state either (a) assumes a conformation other than the chair form, possibly one having a quasi anomeric group, or (b) consists of a mixture of the $\mathrm{CA}$ and $\mathrm{CE}$ conformations. These conclusions agree with Reeves' predirtion for compound 12 (see table 1 ). It should be noted that compound 12 may be an example of a glycoside whose stable conformation is altered by cuprammonia, because, in this solvent, it adopts the CA conformation [8]. Alternatively, it is possible that, for the purpose under consideration, a sugar derivative having the manno configuration should not be compared with related derivatives having the gulo configuration.

All of the foregoing deductions are compatible with Reeves' assignments. Consequently, the validity of the deductions (and the reliability of the method of analysis of the spectra) was apparently established. The spectra of the remaining glycosides (compounds $5,10,11,15,17$, and 19) were, therefore, examined in order to assign an anomeric disposition to each. No prediction has been made as to the stable conformation for each of these glycosides.

A comparison of the spectrum of methyl D-glycero$\alpha$-L-gluco-heptopyranoside (compound 5) with those of the anomers of methyl D-glucopyranoside (compounds 3 and 4 ) indicated its resemblance to the spectrum of methyl $\alpha$-D-glucopyranoside (compound $3)$; see column $\mathrm{C}$ of table 7 . If Reeves' prediction that the most stable conformation for compound 3 is the $\mathrm{CA}$, this observation suggests that compound 5 has the CA conformation in the crystalline state.

The spectrum of methyl 6 -deoxy- $\beta$-L-mannopyranoside (compound 10) was compared with those of its $\alpha$ anomer (compound 9) and of methyl $\alpha$-Dmannopyranoside (compound 8). The results are given in table 8 ; column $\mathrm{C}$ records three bands shown by compounds 8 and 10 that are not shown by compound 9 . However, column D (table 8 ) reveals that the two $\alpha$ anomers have some 13 bands in common that are not exhibited by compound 10 . Since these two $\alpha$ anomers have the $\mathrm{CA}$ conformation as their predicted stable conformation [8], these results suggest that compound 10 has an equatorial anomeric group.

The spectrum of methyl D-glycero- $\alpha$-L-manno-heptopyranoside (compound 11) was now compared with the spectra of compounds 8,9 , and 10 . The results are given in table 9 , except that, to avoid repetition of much of the information in table 8 , the bands common to all four of these glycosides are omitted. Column A (table 9) records three bands shared by compound 11 and methyl 6 -deoxy- $\beta$-Lmannopyranoside (compound 10); column B gives 17 bands that are shown by compound 11 and also by methyl $\alpha$-D-mannopyranoside (compound 8) or methyl 6-deoxy- $\alpha$-L-mannopyranoside (compound 9), or both. Since the predicted stable conformation for the latter two compounds is the CA conformation, this evidence indicates that the anomeric group of compound 11 is axial.

The spectra of the anomers of methyl D-glyceroD-gulo-heptopyranoside [compounds $15(\alpha)$ and 17 $(\beta)$ ] were compared with the spectrum of methyl

TABLE 6. Comparison a of the absorption bands $\left(\mathrm{cm}^{-1}\right)$ shown by the anomers of methyl D-gulopyranoside (12 and 14) and by methyl $\alpha$-D-mannopyranoside (8)

\begin{tabular}{|c|c|c|c|c|c|c|c|c|c|}
\hline \multicolumn{3}{|c|}{ A } & \multicolumn{2}{|c|}{ B } & \multirow{2}{*}{$\frac{\mathrm{C}}{12}$} & \multicolumn{2}{|c|}{ D } & \multicolumn{2}{|c|}{$\mathrm{E}$} \\
\hline 8 & 12 & 14 & 12 & 14 & & 8 & 12 & 8 & 14 \\
\hline 3460 . & 3484 & 3413 & 3257 & 3247 & 3195 & 2841 & 2849 & 1389 & 1385 \\
\hline 3289. & 3333 & 3322 & 2924 & 2915 & 1484 & 1372 & 1372 & 1350 & 1350 \\
\hline 3012 & 3030 & 2976 & 2725 & 2762 & 1437 & 1361 & 1361 & 1258 & 1258 \\
\hline 2950 & 2950 & 2941 & 1302 & 1305 & 1144 & (420?) & (421?) & 1041 & 1036 \\
\hline 2907 & 2907 & 2915 & 1289 & 1289 & 1133 & 412 & (416?) & b 916 & 913 \\
\hline 2841. & 2849 & 2865 & 1276 & 1272 & & (386?) & 378 & b 848 & 855 \\
\hline 1473. & 1471 & 1473 & 1087 & 1087 & & & & (645?) & (656?) \\
\hline 1451 & 1451 & 1449 & 1018 & 1016 & & & & (329?) & 318 \\
\hline 1414 & 1418 & 1418 & 468 & 473 & & & & (304?) & 296 \\
\hline 1399 & 1406 & 1393 & $(360 ?)$ & 360 & & & & 286 & (277?) \\
\hline 1330 & 1333 & 1337 & $(354 ?)$ & (352?) & & & & & \\
\hline 1312 & 1318 & 1316 & & & & & & & \\
\hline $1250 \ldots$ & 1250 & 1258,1238 & & & & & & & \\
\hline $1220^{\circ}$ & 1225 & 1220 & & & & & & & \\
\hline $1199 \mathrm{c}$ & 1193 & 1205 & & & & & & & \\
\hline 1163 & 1159 & 1151 & & & & & & & \\
\hline 1119 & 1121 & 1126 & & & & & & & \\
\hline 1104 & 1096 & 1105 & & & & & & & \\
\hline $1071^{\mathrm{c}}$ & 1078,1070 & 1070 & & & & & & & \\
\hline $1055^{\mathrm{c}}$ - & 1053 & 1056 & & & & & & & \\
\hline 1029 & 1029 & 1026 & & & & & & & \\
\hline $973 \mathrm{~b}$ & 968 & 994 & & & & & & & \\
\hline 890 b_ & 873,872 & 903 & & & & & & & \\
\hline 817 b. & 820 & 798 & & & & & & & \\
\hline $723 \ldots$ & 739 & 751 & & & & & & & \\
\hline $672 \ldots$ & 687 & 675 & & & & & & & \\
\hline 608 & 608 & 601 & & & & & & & \\
\hline $576 \ldots$ & 548 & 553 & & & & & & & \\
\hline 512 & $(506 ?)$ & 506 & & & & & & & \\
\hline 483 & 486 & 490 & & & & & & & \\
\hline $455 \ldots$ & 458 & 444 & & & & & & & \\
\hline $338 \ldots$ & 345 & 333 & & & & & & & \\
\hline
\end{tabular}

a Key: A. Bands shown by methyl $\alpha$-D-mannopyranoside and by both anomers of methyl D-gulopyranoside. B. Bands shown by the methyl D-gulopyranosides, but not by compound 8 . C. Bands shown by methyl $\alpha$-D-gulopyranoside, but not by compounds 8 or 14 . D. Bands shown by methyl $\alpha$-D-mannopyranoside and by methyl $\alpha$-D-gulopyranoside, but not by compound 14 . E. Bands shown by methyl $\alpha$-D-1

b See footnote $a$ to table 3.

c See footnote $b$ to table 5 . 
TABLE 7. Comparison a of ahsorption bands $\left(\mathrm{cm}^{-1}\right)$ shown by the anomers of methyl D-glucopyranoside ( 3 and 4) and by methyl D-glycero- $\alpha$-L-gluco-heptopyranoside (5)

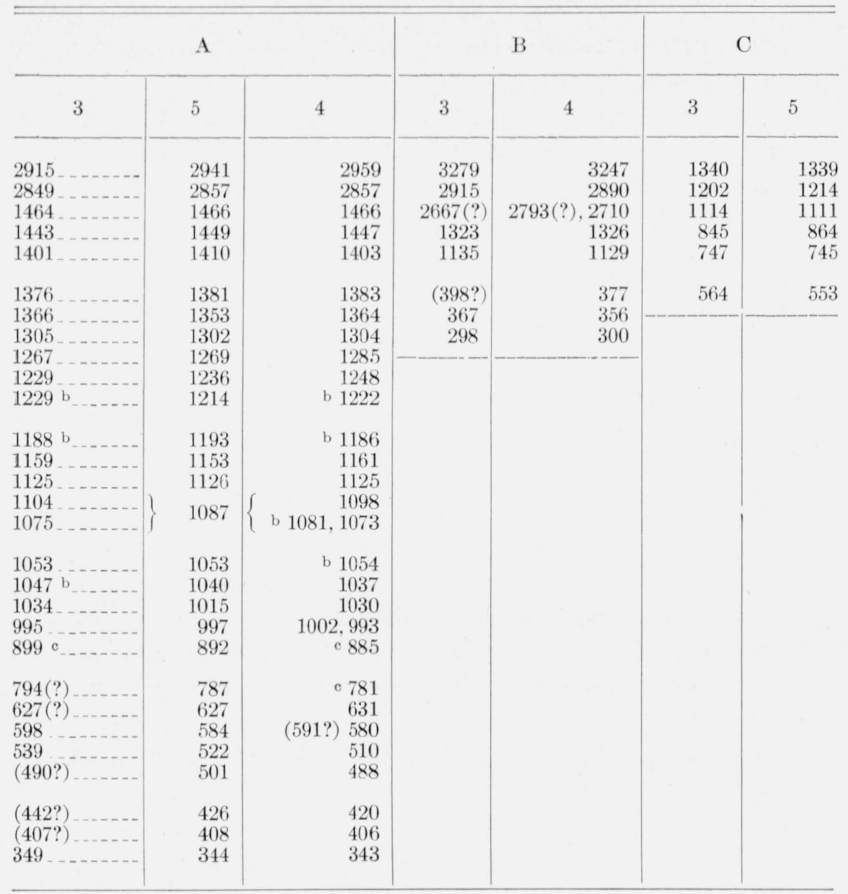

a Key: A. Bands shown by the anomers of methyl D-glucopyranoside and by methyl D-glycero- $\alpha$-L-gluco-heptopyranoside. B. Bands shown by the anomers methyl D-glycero- $\alpha$-L-gluco-heptopyranoside. B. Bands shown by the anomers methyl $\alpha$-D-glucopyranoside and by methyl D-glycero- $\alpha$-L-gluco-heptopyranoside, methyl $\alpha$-D-glucopyranosict

b see footnote $b$ to table 5 .

- See footnote $a$ to table 3 .

$\beta$-D-gulopyranoside (compound 14). Compound 14 showed a band at $751 \mathrm{~cm}^{-1}$; in the same region, compound 15 showed bands at 764 and $738 \mathrm{~cm}^{-1}$. In contrast, compounds 14 and 17 share 17 bands not shown by compound 15 (see column $\mathrm{C}$ of table 10). If the CA conformation predicted as being the stable conformation for compound 14 is accepted, these results suggest that the anomeric group is equatorial in compound 17 and nonequatorial (that is, axial or quasi) in compound 15 .

Finally, the spectrum of cyclohexyl D-glycero- $\beta$ D-gulo-heptopyranoside (compound 19) was studied in comparison with the spectra of compounds 15 and 17 (the $\alpha$ and $\beta$ anomers of the corresponding methyl glycoside). To avoid repetition of much of the information in table 10, the bands common to all three of these glycosides are not given here. The spectrum of compound 15 lacked the following bands in the spectra of compound 17 and (in parentheses) of compound 19: 3509 (3484); 3448 (3436); 2732? (2653?); 1330 (1333); 1311 (1304); 1287 (1287); 1212 (1209); 1122 (1129); 1056 (1056); 1006 (1006); 801 (808); $599(594)$; and $575(575) \mathrm{cm}^{-1}$. If the previous finding is accepted [namely, that the anomeric group of methyl D-glycero- $\beta$-D-gulo-heptopyranoside (compound 17) is equatorial, but that that of the $\alpha$ anomer, compound 15 , is not], these results suggest that the anomeric group of cyclohexyl D-glycero- $\beta$ D-gulo-heptopyranoside is equatorial.
For reasons discussed in section 5.1, the spectra of compounds 13, 16, and 18 were not further examined.

\subsection{Other Absorption Bands}

All of the spectra were studied in regard to the other features listed in table 2 . The hydrates (group 9) showed a band at 1664 to $1634 \mathrm{~cm}^{-1}$.

Compound 19, having a cyclohexyloxy group, showed bands at 2933,2890 , and $2857 \mathrm{~cm}^{-1}$, possibly characteristic of $-\mathrm{CH}_{2}-(\mathrm{C}-\mathrm{H}$ stretching $)$. It also showed bands at 1449 and $1441 \mathrm{~cm}^{-1}$, possibly attributable to $-\mathrm{CH}_{2}-(\mathrm{C}-\mathrm{H}$ deformation $)$.

All of the compounds showed at least one band in the region of 3413 to $3279 \mathrm{~cm}^{-1}$ (associated alcoholic - O-H stretching); at 3012 to $2915 \mathrm{~cm}^{-1}$ (C-H stretching); at 1466 to $1441 \mathrm{~cm}^{-1}$, and at 1346 to $1316 \mathrm{~cm}^{-1}(\mathrm{C}-\mathrm{H}$ bending $)$; and at 1267 to 1235 $\mathrm{cm}^{-1}(\mathrm{C}-\mathrm{O})$. Except for compound 15, all of the compounds showed at least one band in the region of 1247 to $1211 \mathrm{~cm}^{-1}$ (C-O stretching). Compounds $3,6,8,13$, and 20 showed a band at 3289 to 3279 $\mathrm{cm}^{-1}$ (H bonding?).

As previously mentioned, all of the methyl glycopyranosides (group 11 of table 2) show a band in

TAвLE 8. Comparison a of absorption bands $\left(\mathrm{cm}^{-1}\right)$ shown by methyl $\alpha$-D-mannopyranoside (8) and by both anomers of methyl 6-deoxy-L-mannopyranoside (9 and 10)

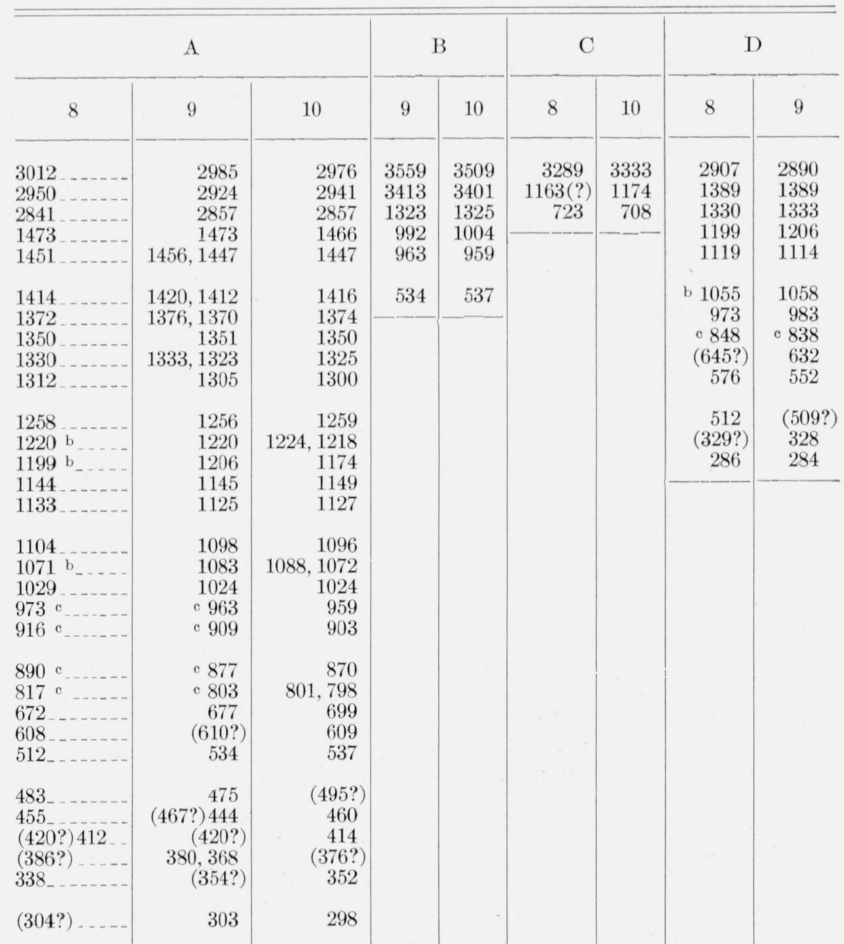

a Key: A. Bands shown by methyl $\alpha$-D-mannopyranoside and by both anomers of methyl 6-deoxy-L-mannopyranoside. B. Bands shown by both anomers of of methyl 6-deoxy-L-mannopyranoside. B. Bands shown by both anomers of methyl 6-deoxy-L-mannopyranoside, but not by compound 8 . C. Bands shown by methyl $\alpha$-D-mannopyranoside and by methyl 6 -deoxy- $\beta$-L-mannopyranoside, but not by compound 9 . D. Bands shown by methyl $\alpha$-D-mannopyr
by methyl 6 -deoxy- $\alpha$-L-mannopyranoside, but not by compound 10 .

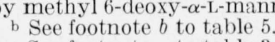

- See footnote $a$ to table 3 . 
TABLE 9. Comparison a of absorption bands $\left(\mathrm{cm}^{-1}\right)$ shown by methyl $\alpha$-D-mannopyranoside (8), the anomers of methyl 6-deoxy-L-mannopyranoside $(9$ and 10$)$, and methyl Dglycero- $\alpha$-L-manno-heptopyranoside (11)

\begin{tabular}{|c|c|c|c|c|}
\hline \multicolumn{2}{|l|}{ A } & \multicolumn{3}{|c|}{ B } \\
\hline 10 & 11 & 8 & 9 & 11 \\
\hline $\begin{array}{l}3333 \\
2710 \\
1287\end{array}$ & $\begin{array}{l}3322 \\
2703(?) \\
1285\end{array}$ & $\begin{array}{c}3460 \\
2907 \\
1399,1389 \\
1361 \\
1250 \\
\text { b } 1199 \\
1119 \\
\text { b } 1055 \\
1041 \\
\text { c } 973 \\
\text { c } 848 \\
(645 ?) \\
576 \\
512 \\
(329 ?) \\
286\end{array}$ & \begin{tabular}{c|}
2890 \\
1389 \\
1342 \\
1206 \\
1114 \\
1058 \\
-983 \\
e 838 \\
632 \\
552 \\
$(509 ?)$ \\
328 \\
284
\end{tabular} & $\begin{array}{r}3425 \\
2915 \\
1395 \\
1368 \\
1340 \\
1241 \\
1200 \\
1115 \\
1048 \\
1036 \\
982 \\
827 \\
654 \\
(578 ?) 562 \\
509 \\
324 \\
277\end{array}$ \\
\hline
\end{tabular}

a Key: A. Bands shown by compounds 10 and 11, but not by compounds 8 and 9. B. Bands shown by compounds 8,9 , and 11 , but not by compound 10 .

See footnote $b$ to table 5

- See footnote $a$ to table 3 .

TABLE 10. Comparison a of absorption bands $\left(\mathrm{cm}^{-1}\right)$ shown by methyl $\beta$-D-gulopyranoside (14) and by the anomers of methyl D-glycero-D-gulo-heptopyranoside (15 and 17)

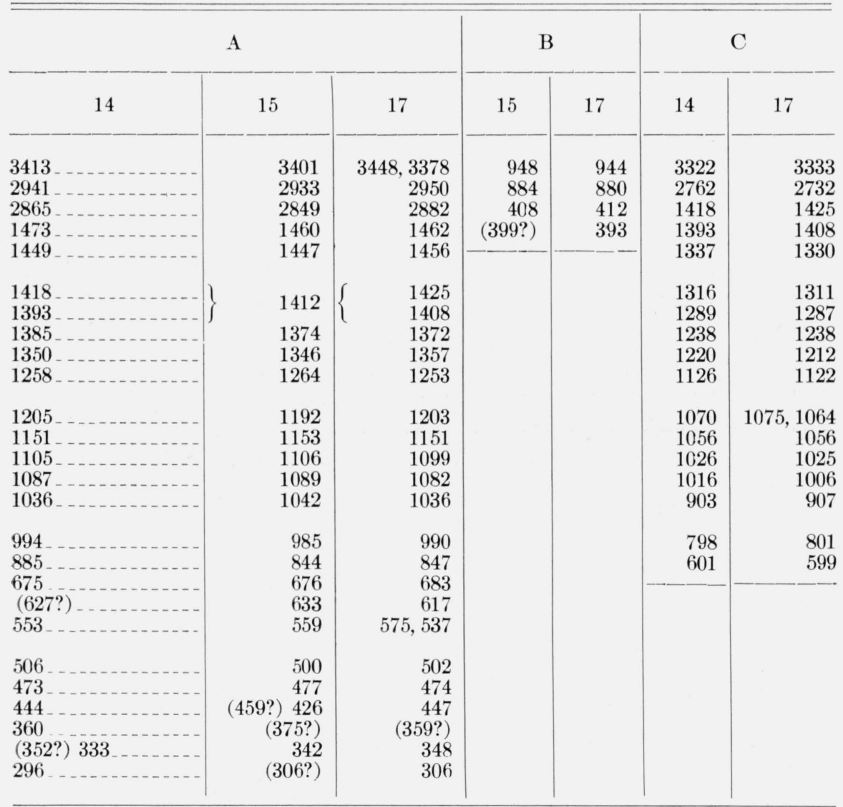

a Key: A. Bands shown by methyl $\beta$-D-gulopyranoside (compound 14) and by both anomers of methyl D-glycero-D-gulo-heptopyranoside. B. Bands shown by both anomers of methyl D-glycero-D-gulo-heptopyranoside, but not by compound 14. C. Bands shown by methyl $\beta$-D-gulopyranoside and by methyl D-glycero- $\boldsymbol{\beta}$-D-gulo-heptopyranoside, but not by compound 15 .

the range of 2882 to $2841 \mathrm{~cm}^{-1}$. This may possibly be attributable to the glycosidic methoxyl group, because Henbest and coworkers [13] have observed that methoxyl groups absorb in the range of 2832 to $2819 \mathrm{~cm}^{-1}$. All of the methyl glycopyranosides also show bands at 1368 to $1330 \mathrm{~cm}^{-1}$, 1285 to $1245 \mathrm{~cm}^{-1}, 1153$ to $1111 \mathrm{~cm}^{-1}$, and 1109 to $1087 \mathrm{~cm}^{-1}$. A band near $1100 \mathrm{~cm}^{-1}$ is characteristic [14] of the methoxyl groups in methoxy-steroids.

\section{Experimental Procedures}

\subsection{Preparation and Purification of the Compounds}

The compounds listed in table 1 were prepared by the methods given in the references cited. Most of the compounds were prepared in the course of an earlier study on the configuration and conformation of methyl glycosides, with reference to optical rotations and rates of hydrolysis [15]. Each substance was recrystallized from an appropriate solvent until further recrystallization caused no change in its melting point or optical rotation.

\subsection{Preparation of the Pellets}

Samples for spectrophotometric study were prepared in the solid phase, as pellets of the crystalline glycoside suspended in an alkali-metal halide, exactly as previously described [4]. For the range of 5000 to $667 \mathrm{~cm}^{-1}$, a concentration of $0.4 \mathrm{mg}$ of glycoside per $100 \mathrm{mg}$ of potassium chloride was used. For the range of 667 to $250 \mathrm{~cm}^{-1}$, the following weights of glycoside per $100 \mathrm{mg}$ of potassium iodide were usedcompounds 1, 2, and 20: $1.33 \mathrm{mg}$; compound 5: (A) 0.33 and (B) $2 \mathrm{mg}$; compound $24: 3 \mathrm{mg}$; and for the rest of the compounds: $2 \mathrm{mg}$. In addition, for the range of 667 to $333 \mathrm{~cm}^{-1}$, the spectrum of compound 12 at a concentration of $2 \mathrm{mg}$ per $100 \mathrm{mg}$ of potassium chloride was recorded. Comparisons of intensity of absorption, from one compound to another, can only be true and quantitative where the molar concentration is the same.

\subsection{Measurement of Infrared Absorption}

The spectrograms are shown in figures 3 and 4 . They were recorded with a Perkin-Elmer Model 21 (double-beam) spectrophotometer equipped with a prism of sodium chloride (for the range of 5000 to $667 \mathrm{~cm}^{-1}$ ) and of cesium bromide (for the range of 667 to $250 \mathrm{~cm}^{-1}$ ), as previously described [4].

Some absorption attributable to water (in the compound, the alkali halide, or both) was observed at 3448 and $1639 \mathrm{~cm}^{-1}$ and, attributable to atmospheric water vapor, in the far-infrared curves. These regions are drawn on the spectrograms with dashed lines which are merely precautionary and are not to be interpreted quantitatively.

\subsection{Spectra Measured Under Different Conditions}

Because of the possibility of interaction of the various compounds with the pelleting halide under high pressure, the spectra of a few of the glycosides, chosen at random, were also recorded in a Nujol mull (requiring no pressure) for comparison. Since a number of these compounds gave markedly different spectrograms in potassium iodide and in Nujol, respectively, the spectra of all of them were now recorded in Nujol. For 16 of the 24 glycosides, the spectra obtained with either medium matched well. However, the following compounds gave spectrograms that were different in Nujol and in potassium iodide: compounds $1,5,7,10,11,12,15$, and 20 . 

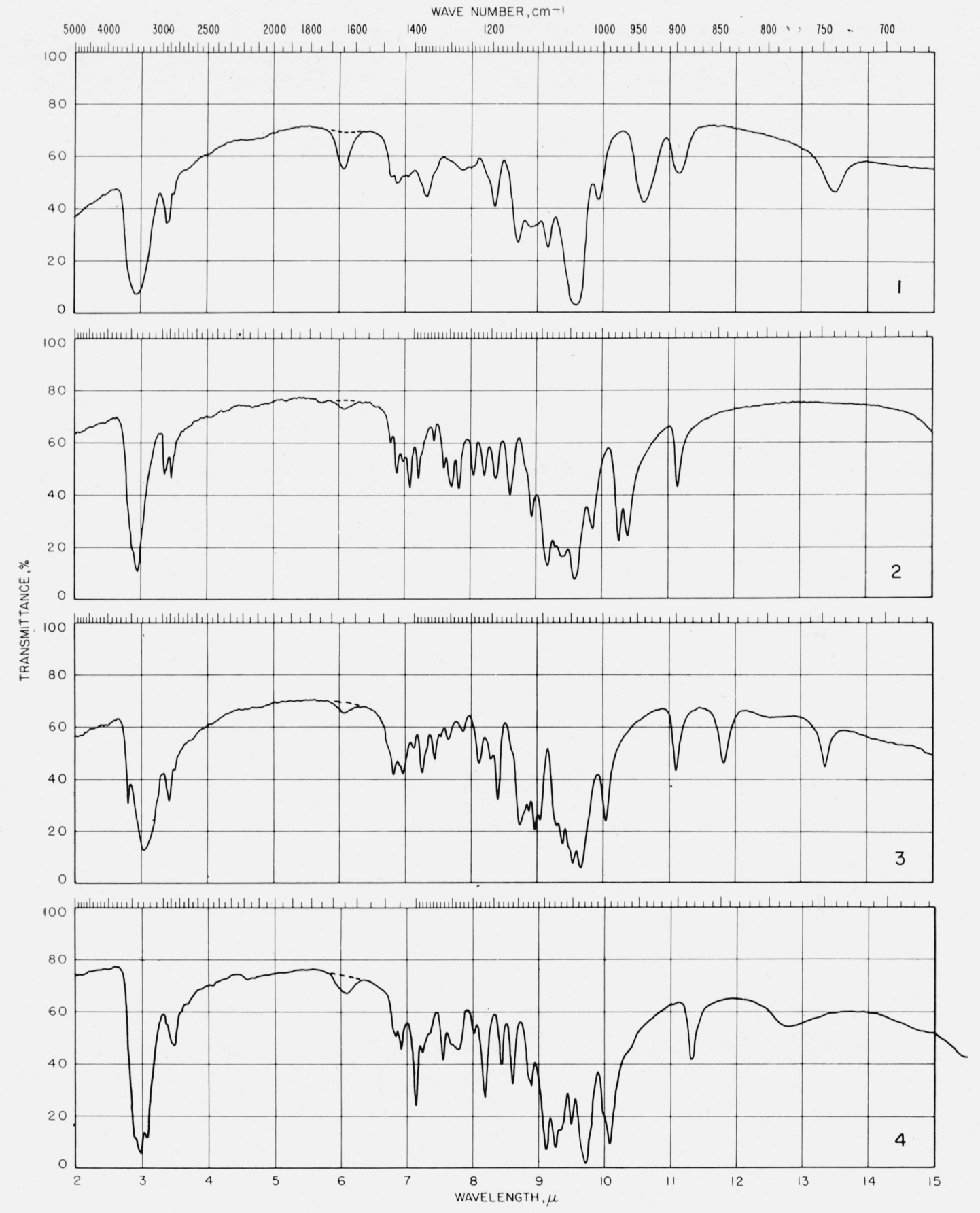

FIGURE 3. Spectrograms of materials in potassium chloride pellets.

1, Methyl $\alpha$-D-xylopyranoside; 2, methyl $\beta$-D-xylopyranoside; 3, methyl $\alpha$-D-glucopyranoside; 4 , methyl $\beta$-D-glucopyranoside. 

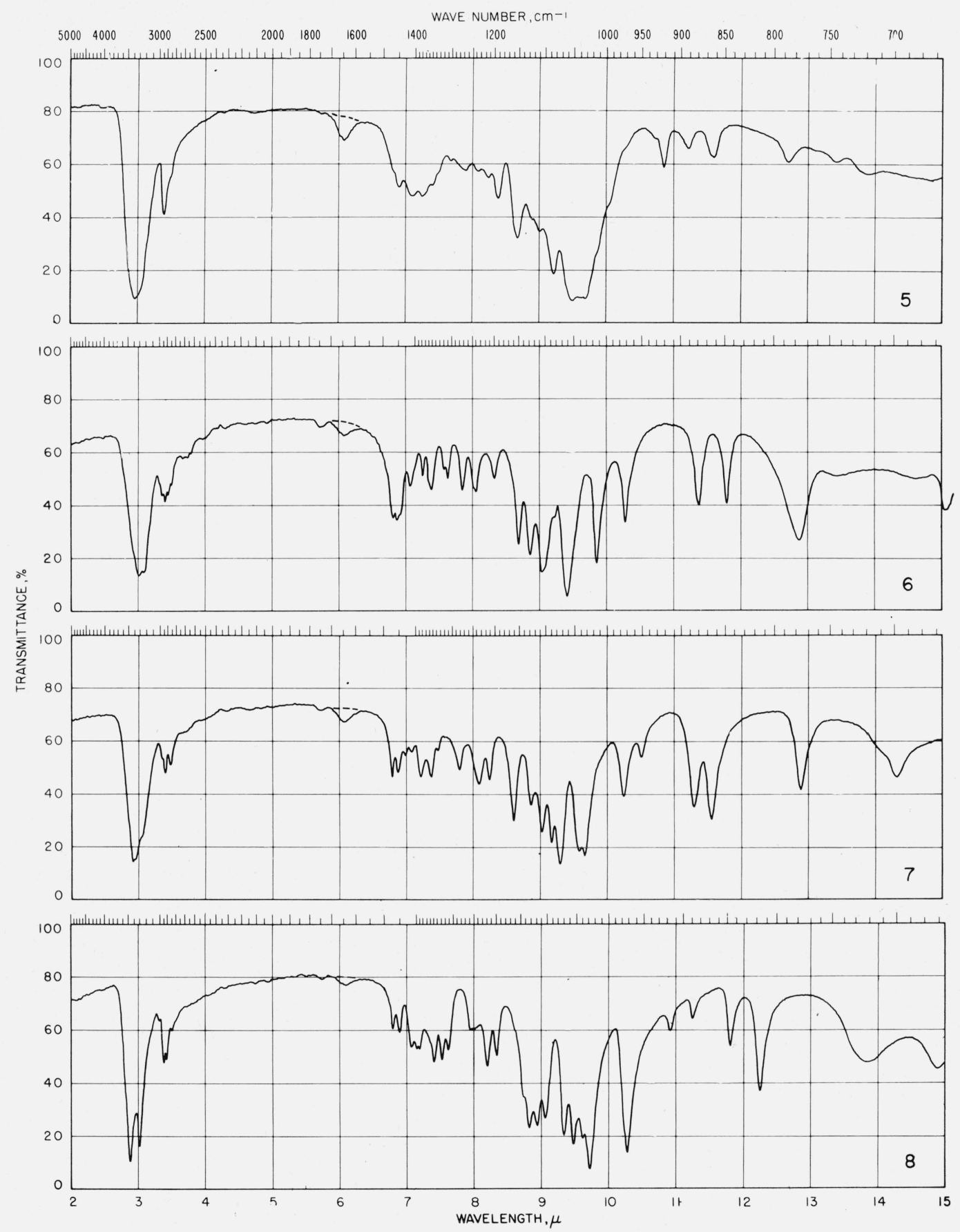

Figure 3. Spectrograms of materials in potassium chloride pellets.-Continued 5 , methyl D-glycero- $\alpha$-L-gluco-heptopyranoside; 6, methyl $\alpha$-D-lyxopyranoside; 7, methyl $\beta$-D-lyxopyranoside; 8 , methyl $\alpha$-D-mannopyranoside. 

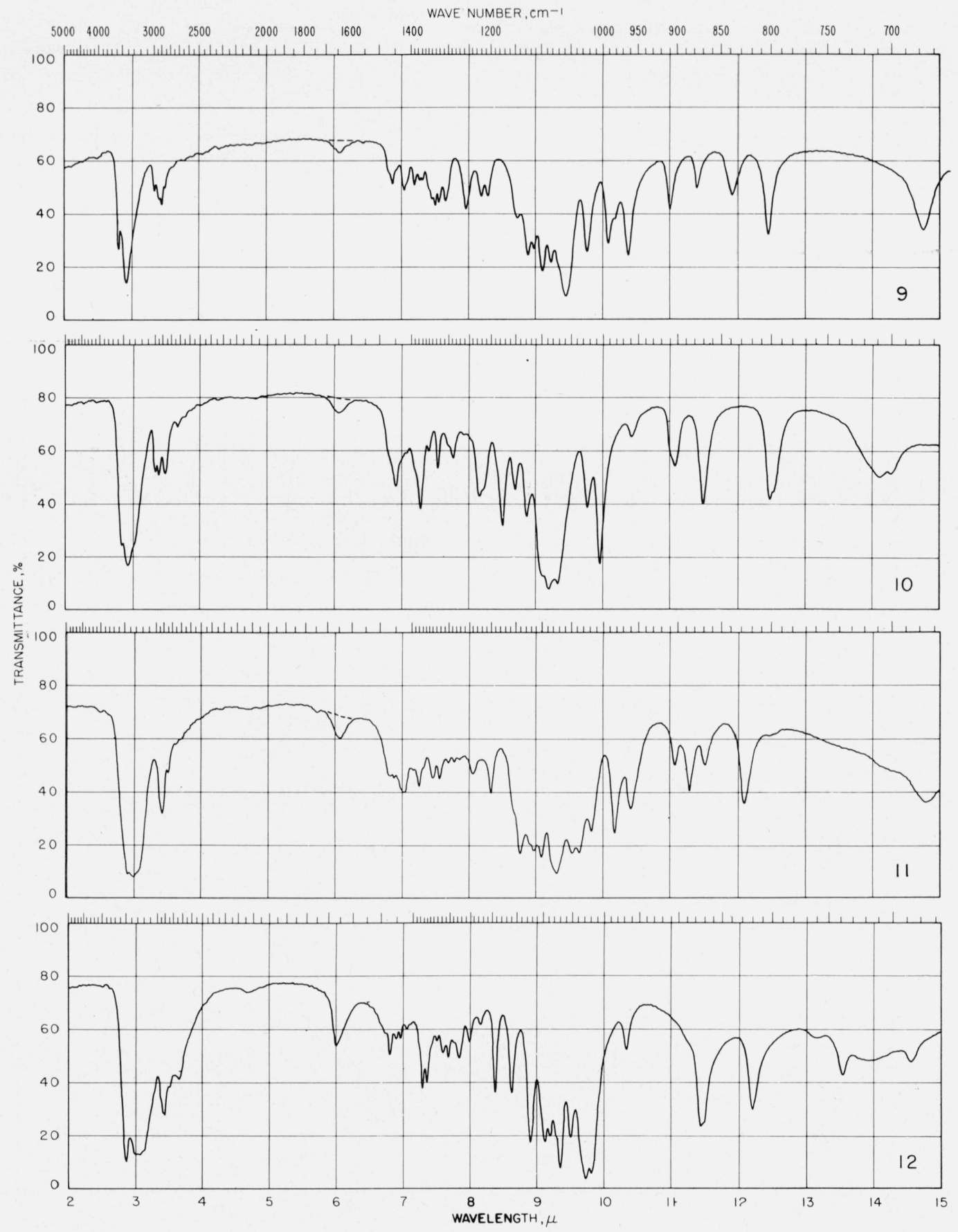

Figure 3. Spectrograms of materials in potassium chloride pellets.-Continued

9, methyl 6-deoxy- $\alpha$-L-mannopyranoside; 10, methyl 6-deoxy- $\beta$-L-mannopyranoside; 11 , methyl D-glycero- $\alpha$-L-manno-heptopyranoside; 12, methyl $\alpha$-D-gulopyranoside monohydrate. 

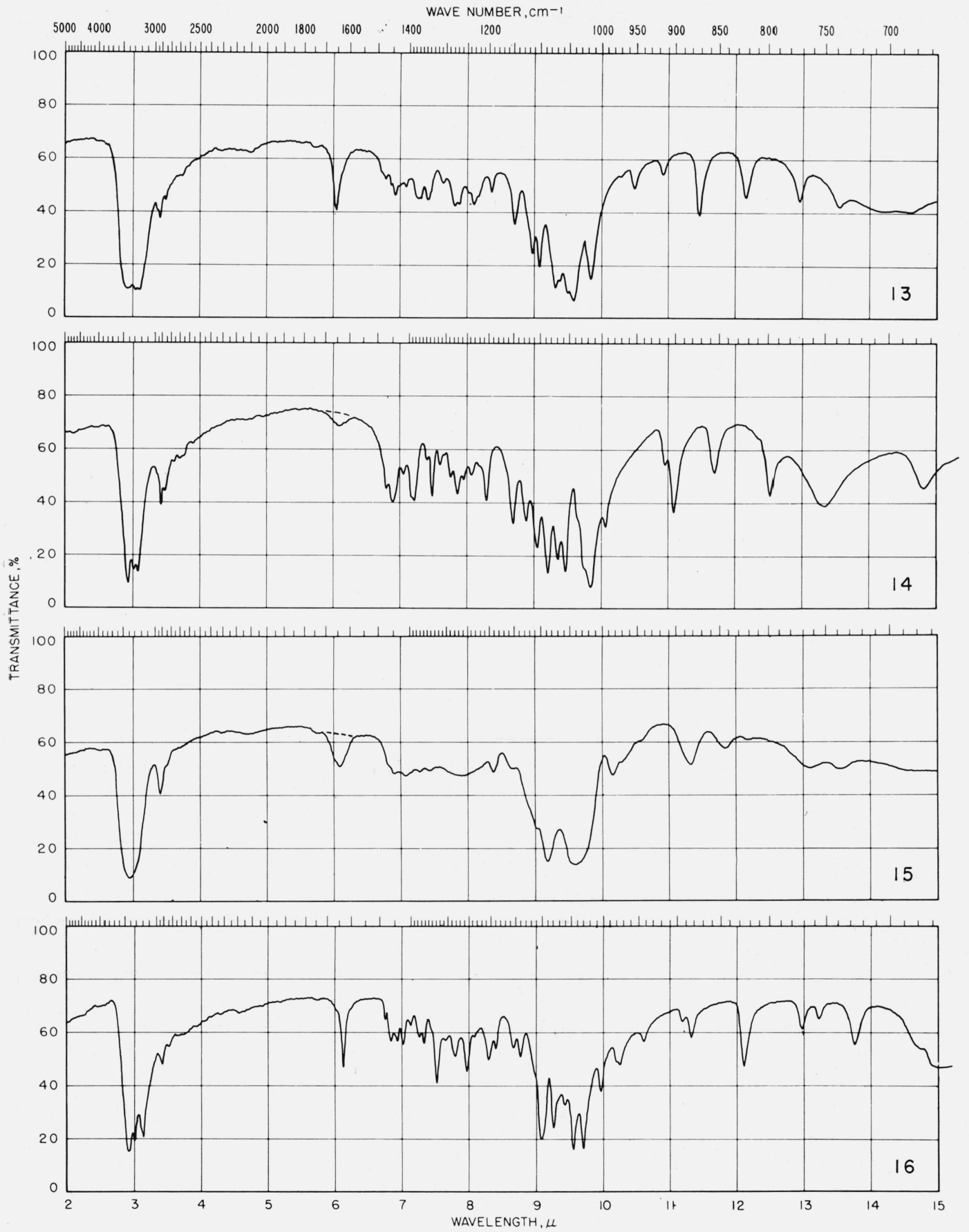

Figure 3. Spectrograms of materials in potassium chloride pellets.-Continued

13, methyl $\alpha$-D-gulopyranoside.1/2 $\left(\mathrm{CaCl}_{2} \cdot 3 \mathrm{H}_{2} \mathrm{O}\right) ; \mathbf{1 4}$, methyl $\beta$-D-gulopyranoside; 15 , methyl D-glycero- $\alpha$-D-gulo-heptopyranoside; 16 , methyl D-glycero- $\alpha$-D-gulo-heptopyranoside $\cdot \mathrm{CaCl}_{2} \cdot \mathrm{H}_{2} \mathrm{O}$. 

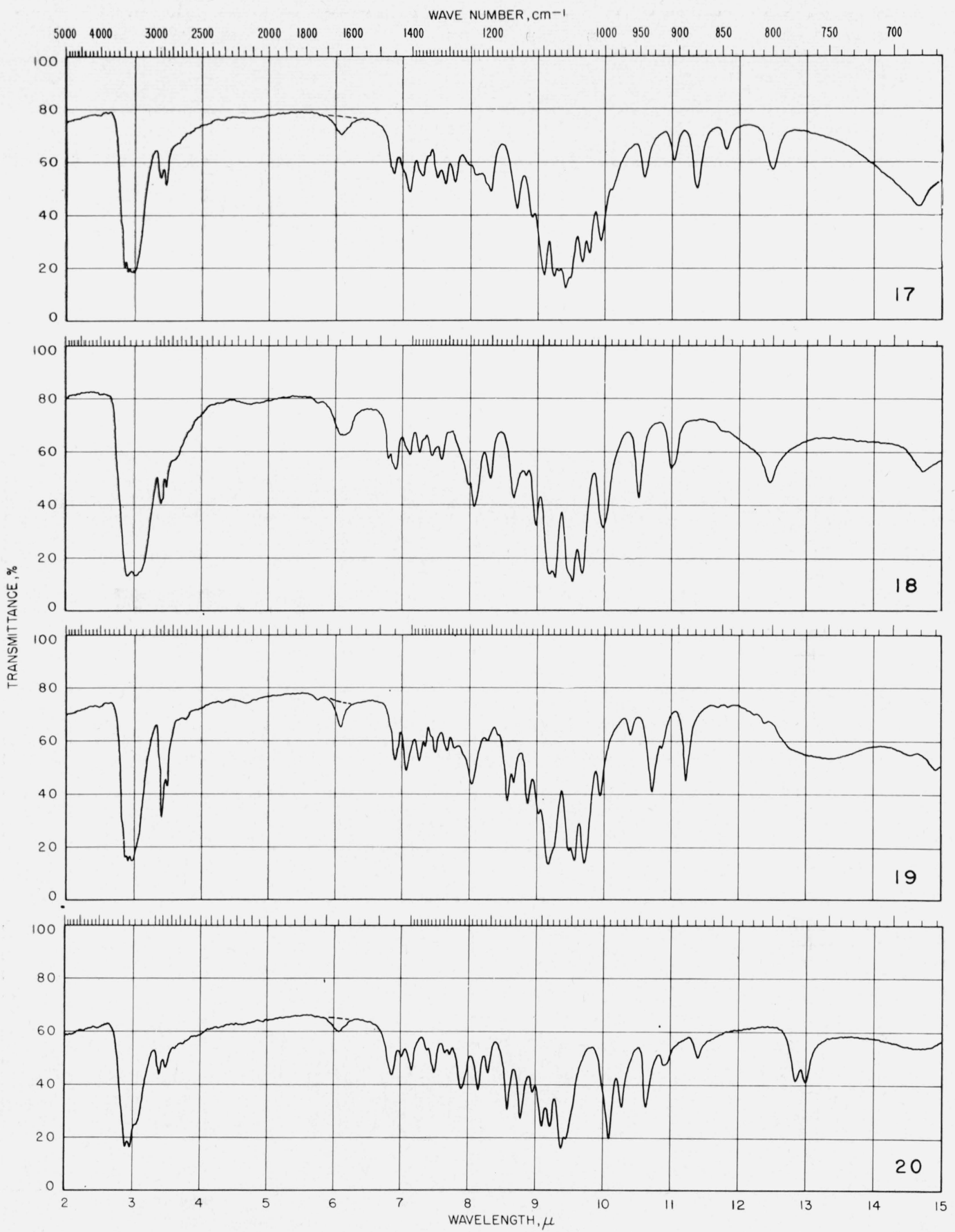

Figure 3. Spectrograms of materials in potassium chloride pellets.-Continued

17 , methyl D-glycero- $\beta$-D-gulo-heptopyranoside; 18 , methyl D-glycero- $\beta$-D-gulo-heptopyranoside $\cdot 1 / 2 \mathrm{CaCl}_{2} \cdot \mathrm{H}_{2} \mathrm{O} ; 19$, cyclohexyl D-glycero$\beta$-D-gulo-heptopyranoside; 20 , methyl $\alpha$-L-arabinopyranoside. 

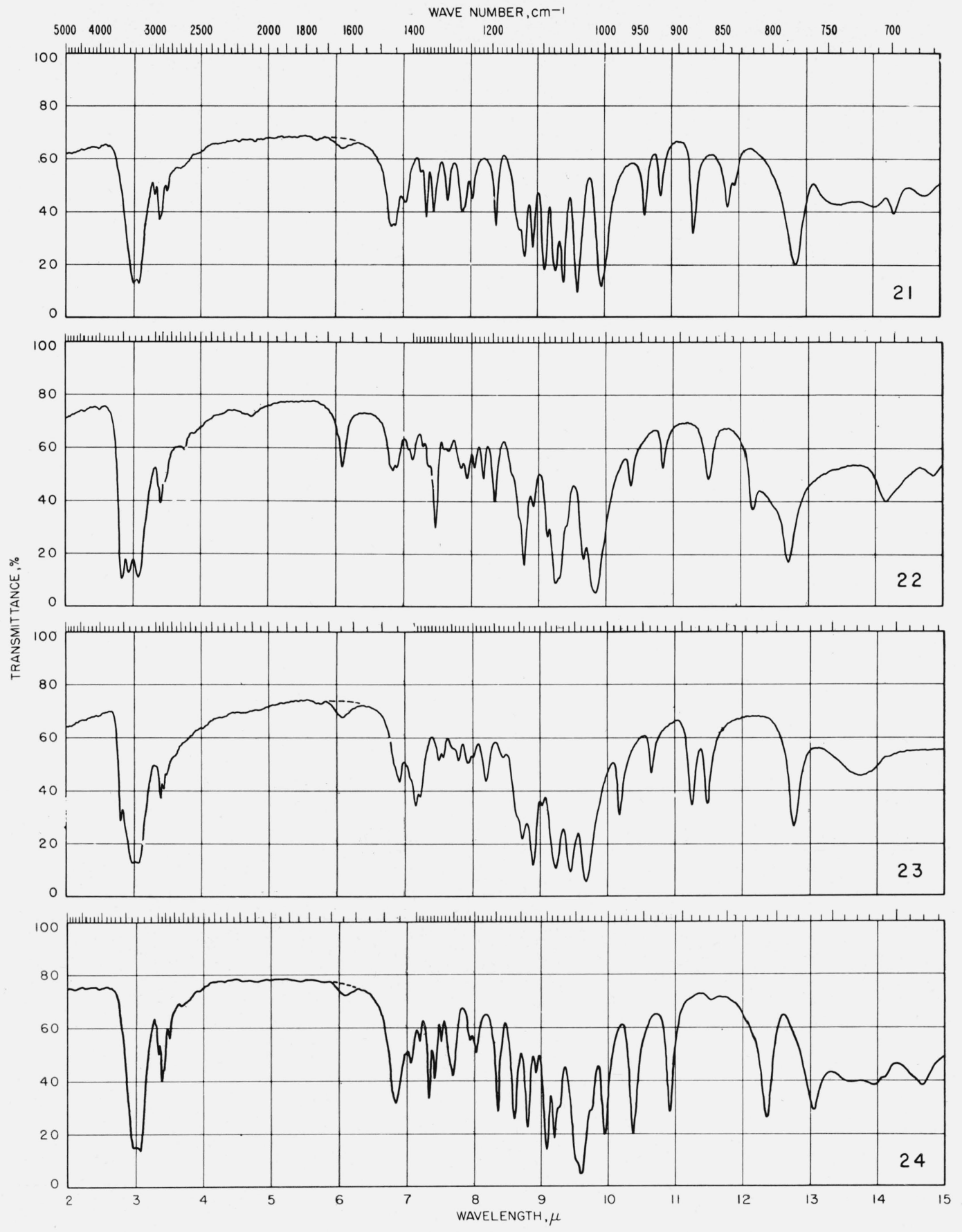

FIGURE 3. Spectrograms of materials in potassium chloride pellets.-Continued

21, methyl $\beta$-L-arabinopyranoside; 22, methyl $\alpha$-D-galactopyranoside monohydrate; 23, methyl $\beta$-D-galactopyranoside; 24, methyl 6-deoxy- $\alpha$-L-galactopyranos:de. 

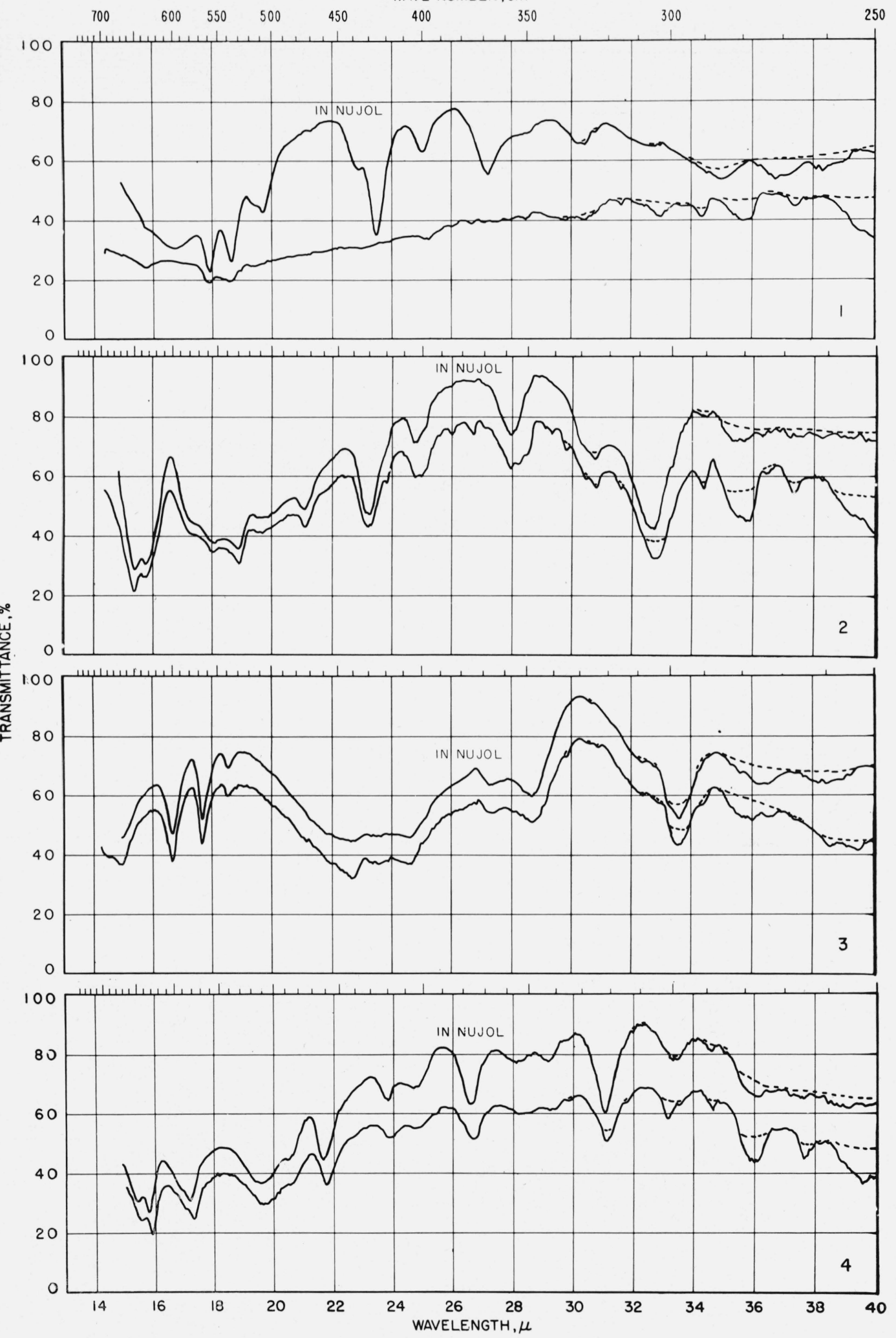

Figure 4. Spectrograms of materials in Nujol mulls and in potassium iodide pellets.

1, Methyl $\alpha$-D-xylopyranoside; 2 , methyl $\beta$-D-xylopyranoside; 3, methyl $\alpha$-D-glucopyranoside; 4 , methyl $\beta$-D-glucopyranoside. 
WAVE NUMBER, $\mathrm{cm}^{-1}$
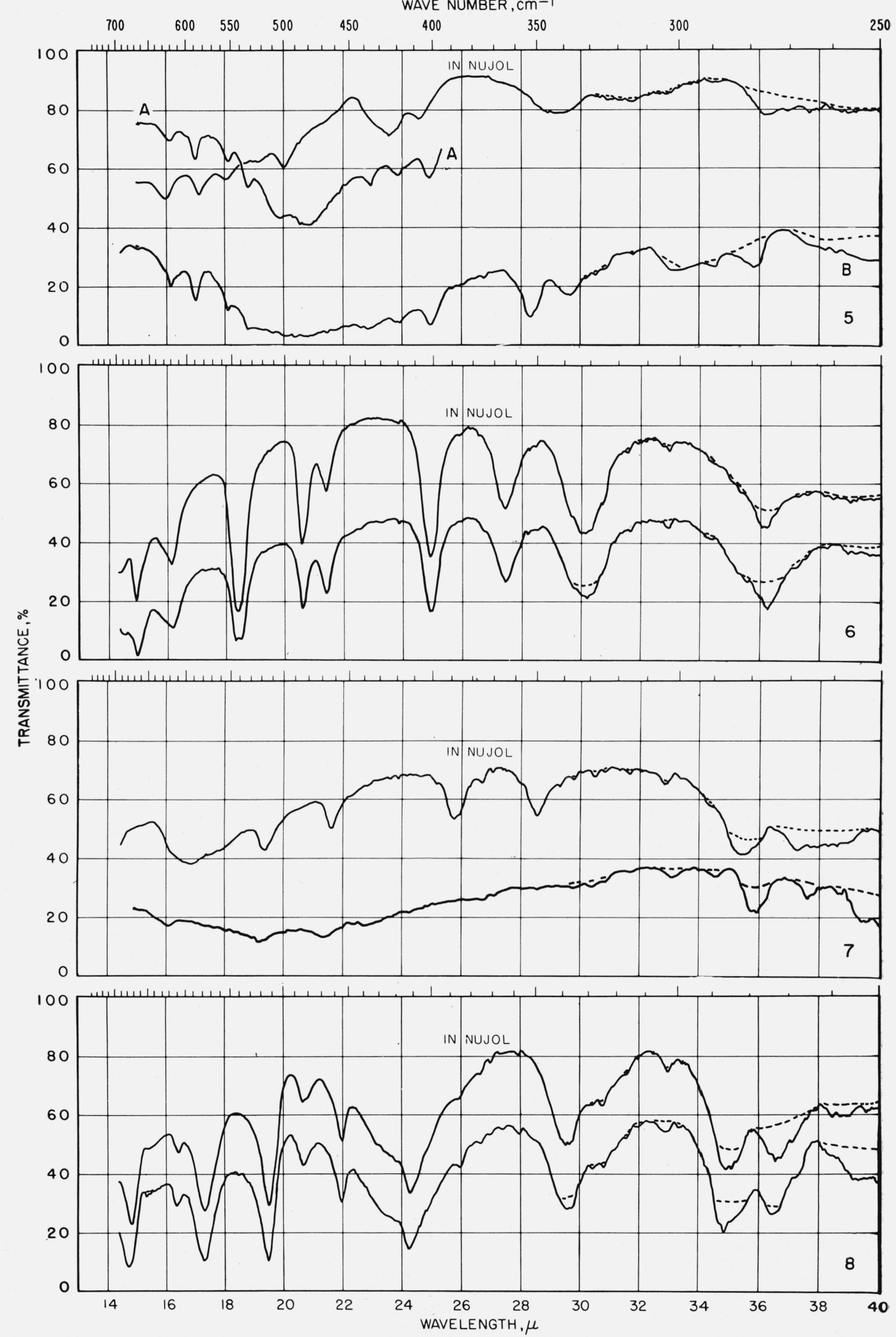

Figure 4. Spectrograms of materials in Nujol mulls and in potassium iodide pellets.-Continued

5 , methyl D-glycero- $\alpha$-L-gluco-heptopyranoside; 6, methyl $\alpha$-D-lyxopyranoside; 7 , methyl $\beta$-D-lyxopyranoside; 8 , methyl $\alpha$-D-mannopyranoside. 
WAVE NUMBER, $\mathrm{cm}^{-1}$
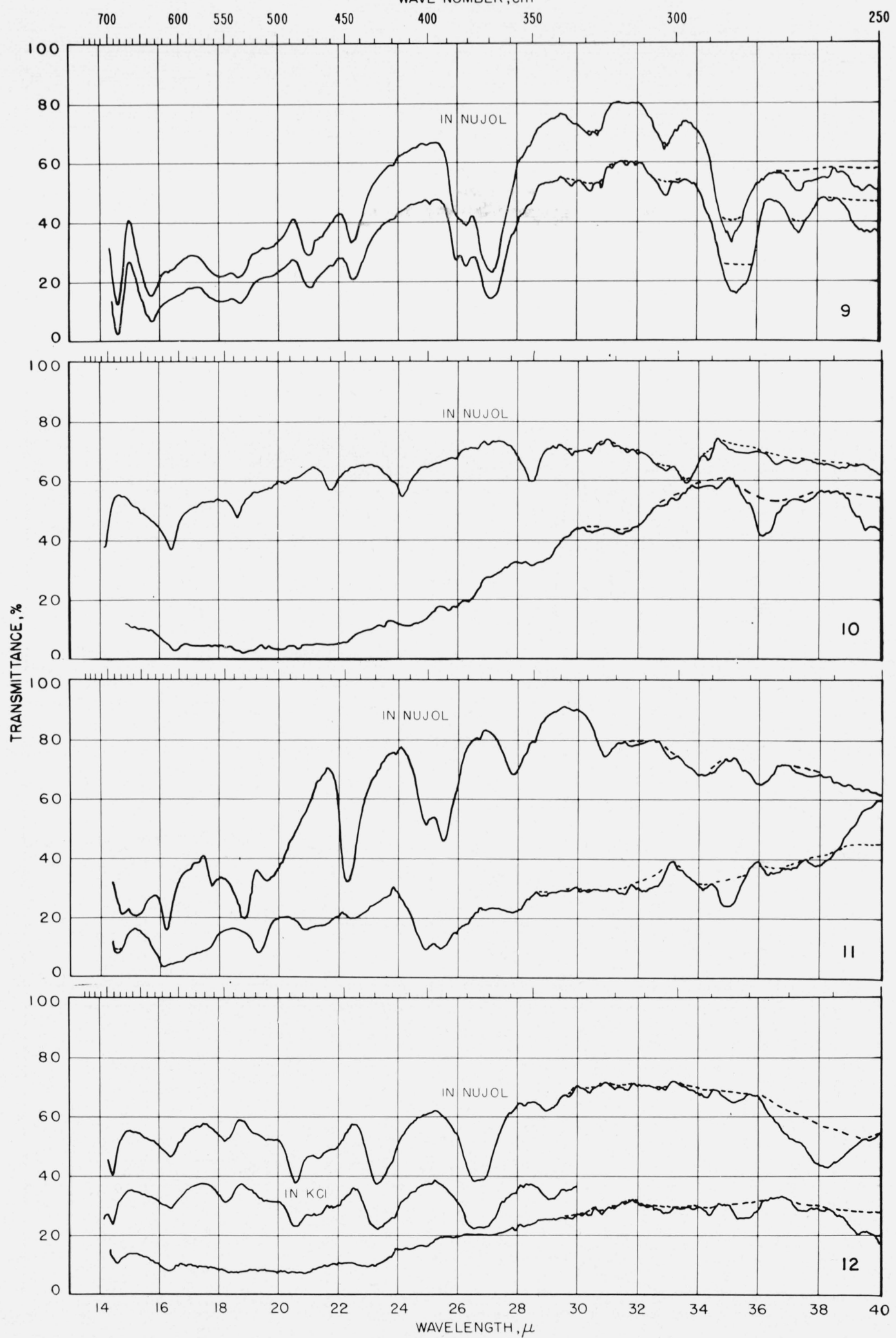

Figure 4. Spectrograms of materials in Nujol mulls and in potassium iodide pellets.-Continued

9, methyl 6-deoxy- $\alpha$-L-mannopyranoside; 10, methyl 6-deoxy- $\beta$-L-mannopyranoside; 11 , methyl D-glycero- $\alpha$-L-manno-heptopyranoside; 12 , methyl $\alpha$-D-gulopyranoside monohydrate. 

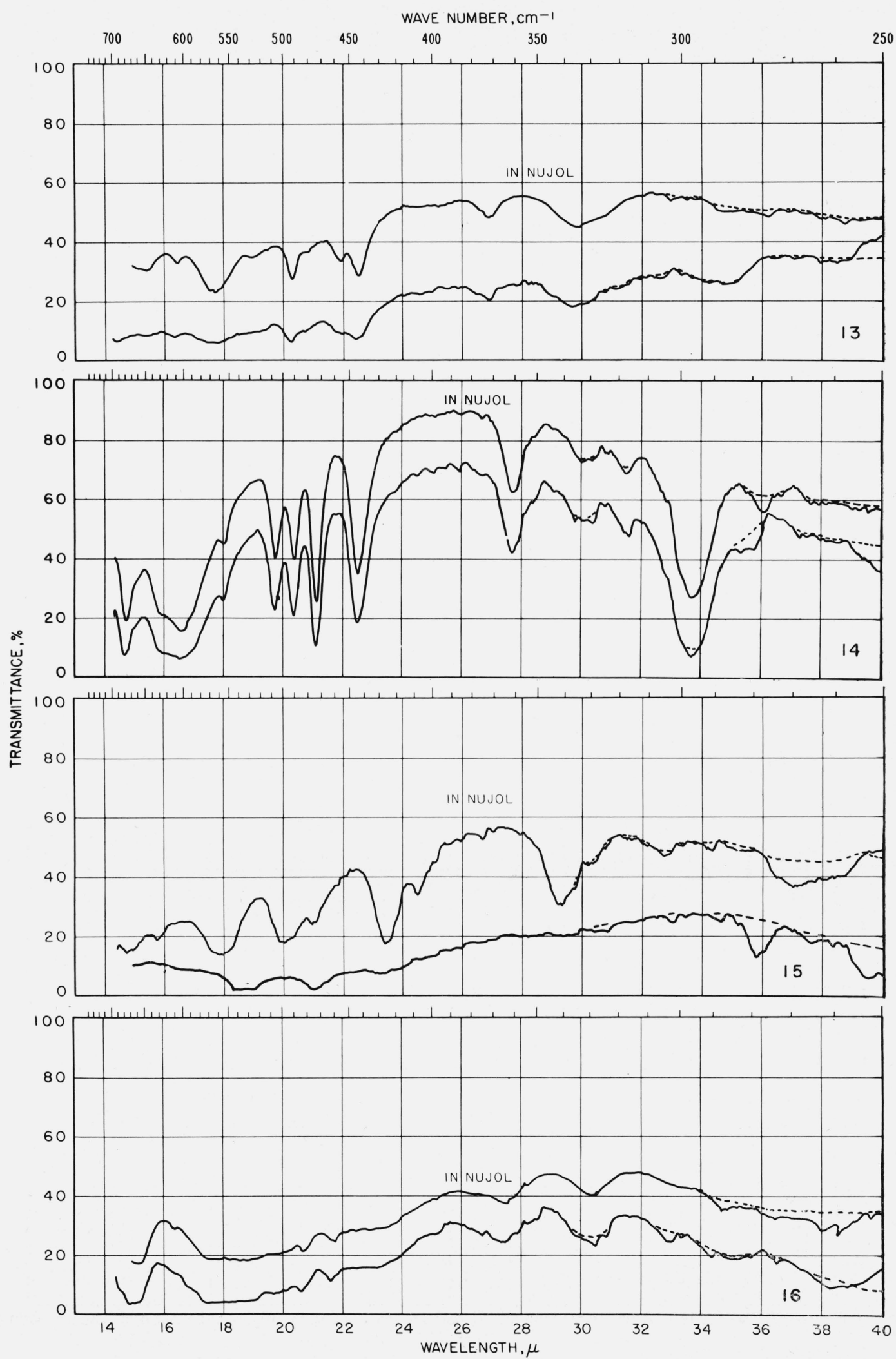

Figure 4. Spectrograms of materials in Nujol mulls and in potassium iodide pellets.-Continued

13, methyl $\alpha$-D-gulopyranoside $\cdot 1 / 2\left(\mathrm{CaCl}_{2} \cdot 3 \mathrm{H}_{2} \mathrm{O}\right) ; 14$, methyl $\beta$-D-gulopyranoside; 15, methyl D-glycero- $\alpha$-D-gulo-heptopyranoside; 16 , methyl D-glycero- $\alpha$-D-gulo-heptopyranoside $\cdot \mathrm{CaCl}_{2} \cdot \mathrm{H}_{2} \mathrm{O}$. 

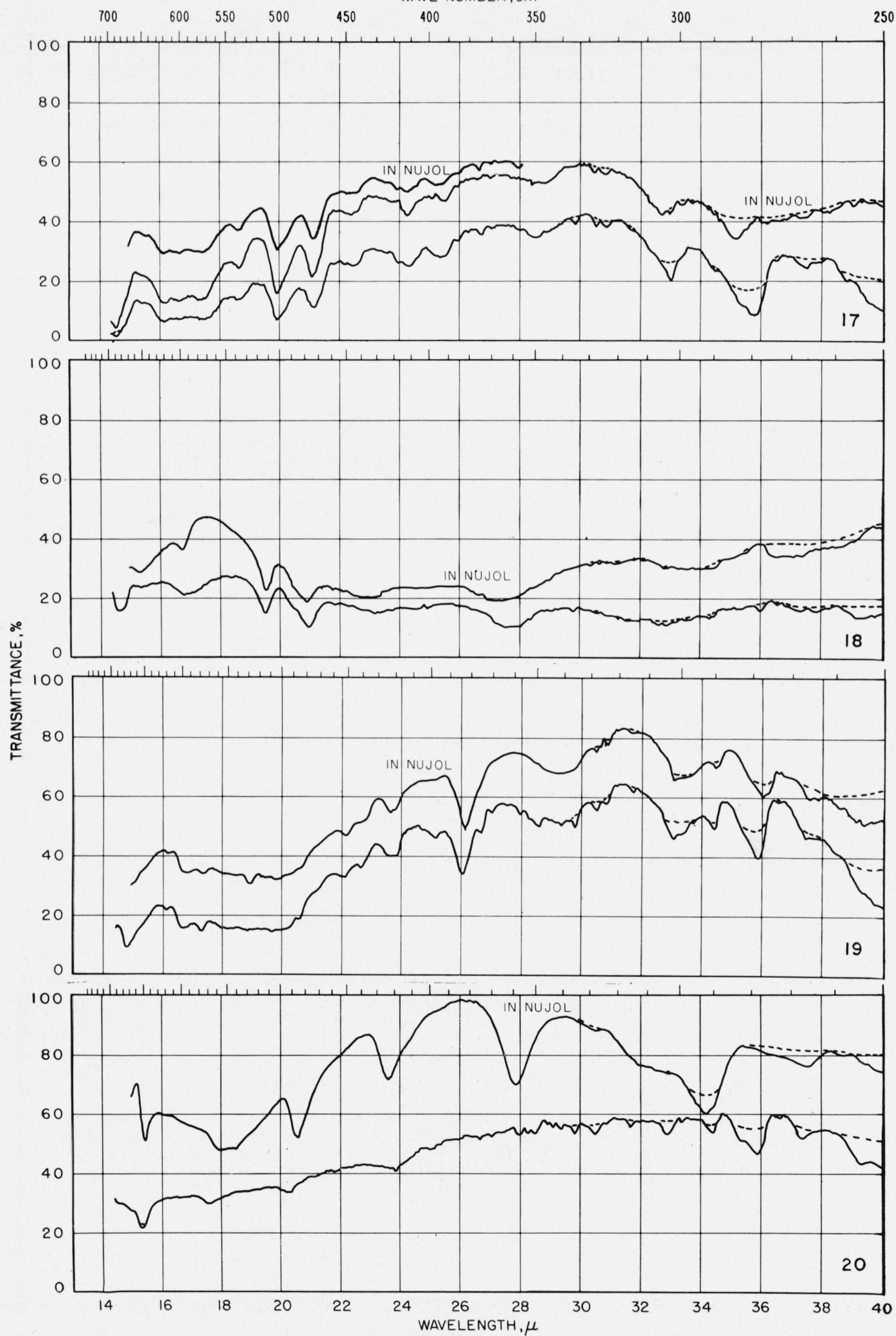

Figure 4. Spectrograms of materials in Nujol mulls and in potassium iodide pellets.-Continued

17, methyl D-glycero- $\beta$-D-gulo-heptopyranoside; 18 , methyl D-glycero- $\beta$-D-gulo-heptopyranoside $\cdot 1 / 2 \mathrm{CaCl}_{2} \cdot \mathrm{H}_{2} \mathrm{O} ; \mathbf{1 9}$, cyclohexyl D-glycero$\beta$-D-gulo-heptopyranoside; 20, methyl $\alpha$-L-arabinopyranoside. 

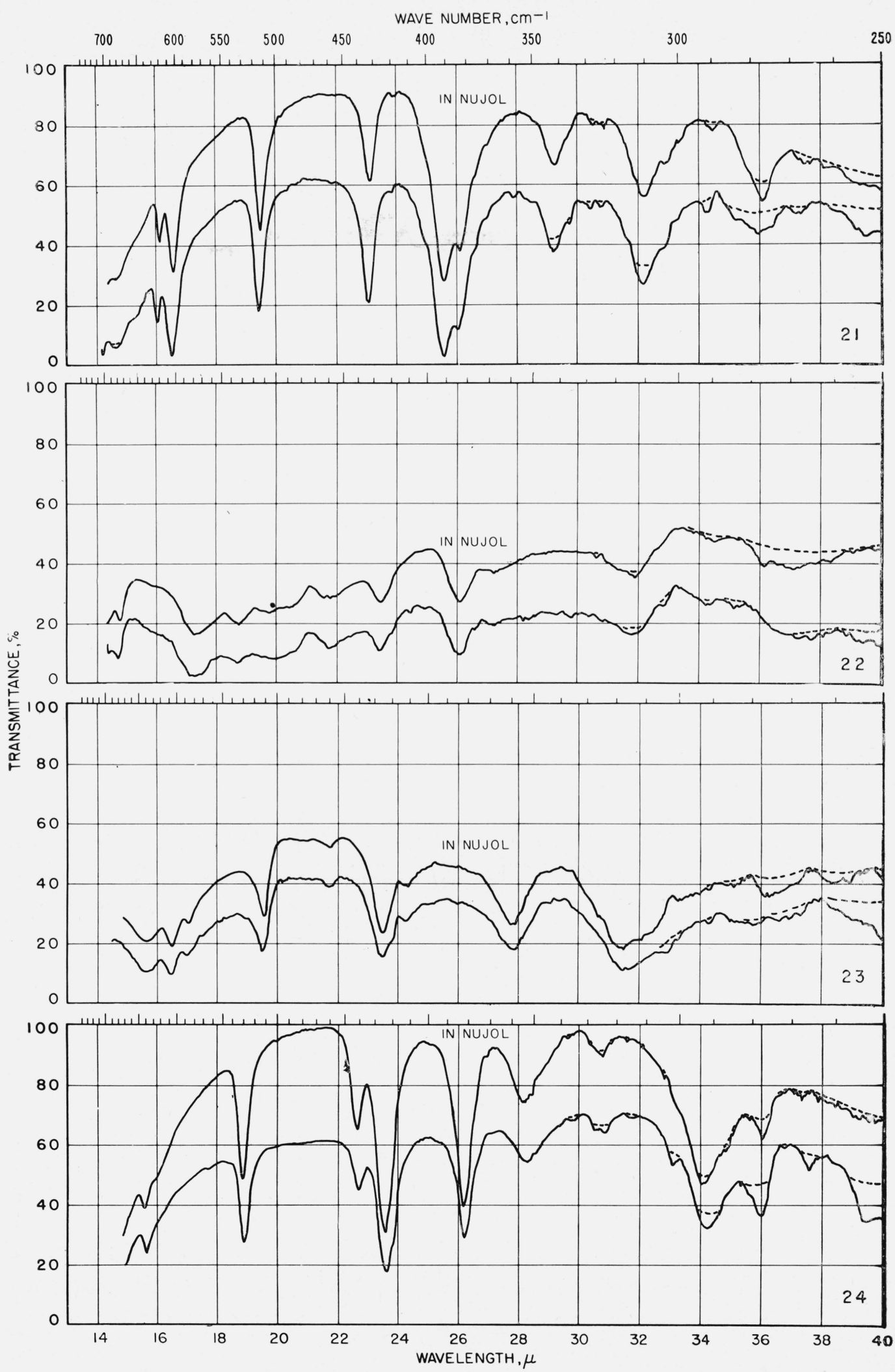

Figure 4. Spectrograms of materials in Nujol mulls and in potassium iodide pellets.--Continued

21, methyl $\beta$-L-arabinopyranoside; 22, methyl $\alpha$-D-galactopyranoside monohydrate; 23, methyl $\beta$-D-galactopyranoside; 24, methyl 6-deoxy- $\alpha$-L-galactopyranoside. 
Of these, compounds 12 and 15 are known to give a molecular complex with calcium chloride, affording compounds 13 and 16 , respectively; both compound 13 and compound 16 give essentially the same spectrum in the two media. This observation might suggest a relationship between the ability to form a complex with calcium chloride and the behavior observed in potassium iodide. However, compound 17 , which also forms a complex with calcium chloride (namely, compound 18), gives, like compound 18, essentially the same spectrum in the two media. A possible explanation is that some glycosides may react with both calcium chloride and potassium iodide, whereas others may react with only one of these salts.

It is not known whether compounds $1,5,7,10$, 11, and 20 form complexes with calcium chloride, nor whether any of the eight glycosides that give an unsatisfactory spectrum in potassium iodide do actually react chemically with this iodide.

In view of these observations, the spectra obtained with a Nujol mull were used exclusively for measuring the positions of absorption bands in the range of 667 to $250 \mathrm{~cm}^{-1}$, not only for the eight glycosides that give unsatisfactory spectra in potassium iodide, but also (in order to keep the measurements strictly comparable) for the 16 other glycosides.

Finally, to make sure that compound 12 (typical of the glycosides "reacting" with both calcium chloride and potassium iodide) does not react with potassium chloride, the spectrum in the range of 667 to 333 $\mathrm{cm}^{-1}$ was recorded for a pellet of compound 12 in potassium chloride and compared with its spectrum in Nujol; the spectra matched well. Consequently, the spectra in potassium chloride in the range of 5000 to $667 \mathrm{~cm}^{-1}$ were accepted as being satisfactory.

The authors express their gratitude to Harriet L. Frush and J. D. Moyer for preparing and purifying many of the compounds used in this study; to Alex Cohen for technical assistance; and to J. E. Stewart, J. J. Comeford, and F. P. Czech for recording the infrared absorption spectra.

\section{References}

[1] H. S. Isbell and R. S. Tipson, Science 130, 793 (1959).

[2] H. S. Isbell and R. S. Tipson, J. Research NBS 64A, 171 (1960).

[3] H. S. Isbell, F. A. Smith, E. C. Creitz, H. L. Frush, J. D. Moyer, and J. E. Stewart, J. Research NBS 59, 41 (1957) RP2772.

[4] R. S. Tipson, H. S. Isbell, and J. E. Stewart, J. Research NBS 62, 257 (1959) RP2960.

[5] H. S. Isbell, J. Research NBS 57, 171 (1956) RP2707.

[6] R. E. Reeves, J. Am. Chem. Soc. 71, 215 (1949).

[7] R. E. Reeves, J. Am. Chem. Soc. \%1, 1737 (1949).

[8] R. E. Reeves, J. Am. Chem. Soc. \%2, 1499 (1950).

[9] R. E. Reeves, Ann. Rev. Biochem. 27, 15 (1958).

[10] G. R. Barker and D. F. Shaw, J. Chem. Soc. 1959, 584.

[11] L. P. Kuhn, Anal. Chem. 22, 276 (1950).

[12] R. E. Reeves, Advances in Carbohydrate Chem. 6, 107 (1951).

[13] H. B. Henbest, G. D. Meakins, B. Nicholls, and A. A. Wagland, J. Chem. Soc. 195\%, 1462.

[14] J. E. Page, J. Chem. Soc. 1955, 2017.

[15] H. S. Isbell and H. L. Frush, J. Research NBS 24, 125 (1940) RP1274.

Washington, D.C.

(Paper 64A3-48) 


\section{Publications of the National Bureau of Standards*}

\section{Selected Abstracts}

\section{Measurement of cobalt-60 and cesium-137 gamma rays with a free-air chamber, $H$. O. Wyckoff, $J$. Research NBS 64C, No. 2, 87 (1960).}

\begin{abstract}
Design data for free-air chambers measuring cobalt-60 and cesium-137 gamma rays in roentgens have been presented. It has been shown that the Jaffee-Zanstra method of obtaining the saturation current is adequate for air pressures of about 4 to 12 atmospheres. Also, radiation measurements of the gamma rays from cobalt-60 and cesium-137 made by a cavity chamber and a free-air chamber agree to within the experimental errors.
\end{abstract}

Radiation field from a rectangular source, J. H. Hubbell, R. L. Bach, and J. C. Lamkin, J. Research NBS 64C, No. 2, 121 (1960).

Many radiation shielding problems involve calculations of the response of an isotropic detector to radiation of arbitrary angular distribution from uniform rectangular sources. In calculations of this type the family of integrals $\int_{S}\left(\cos \theta d S / r^{2}\right)$ $P_{l}(\cos \theta)$ and the integral $\int_{S}\left(d \dot{S} / r^{2}\right) \exp (-\mu t / \cos \theta)$ are frequently encountered, where $\theta$ is obliquity with respect to an axis perpendicular to the plane containing the rectangular radiant surface, $S, r$ is the distance from an element of source area, $d S$, to the detector, $\mu$ is the attenuation coefficient, and $t$ is the barrier thickness. The first type of integral facilitates use of Legendre expansion representations of radiation directional distributions, and may also have application in other radiant surface studies, such as illumination and heat exchange engineering. The second integral relates to unscattered gamma rays from a monoenergetic, plane isotropic, rectangular source separated from the detector by a laver of material of thickness $t$. Formulas, expansions, and numerical results are presented.

Response of a loaded electric dipole in an imperfectly conducting cylinder of finite length, C. W. Harrison, Jr., and R. W. P. King, J. Research NBS 64D, No. 3, 289 (1960).

Analytical relationships are developed which permit calculation of the power of the load impedance of an electric probe, symmetrically located within an imperfectly conducting cylinder of small radius compared to the wavelength, in terms of the electric field incident upon the cylinder.

Basic theorems in matrix theory, M. Marcus, NBS Applied Math. Series 57 (1960) 15 cents.

This is a survey of the basic identities and inequalities of matrix theory. Included are results dealing with elementary properties, canonical forms, invariance, congruence, commutativity, orthogonalization, eigenvalues, determinants, submatrices, rank, determinant and rank inequalities, numerical methods for inversion and eigenvalues, condition numbers.

Paramagnetic resonance in the free hydroxyl radical, H. E. Radford, Nuovo cimento, Serie $X 14,245$ (1959).

Paramagnetic resonance has been observed in electrically discharged water vapor. A calculation of the microwave Zeeman effect for the free $\mathrm{OH}$ radical allows a consistent interpretation of the spectrum as arising from electric dipole transitions within the low-lying rotational levels of $\mathrm{OH}$ The spectrum permits accurate measurements of $g$-factors, $\Lambda$-doubling intervals, and hyperfine structure. Higher order effects of $\Lambda$-doubling on the Zeeman and hyperfine interactions are detectable. Corresponding OD spectra have been observed in discharged $\mathrm{D}_{2} \mathrm{O}$.

Relaxation processes in multistate systems, K. E. Shuler, Phys. of Fluids 2, No. 4, 442 (1959).

An analysis is made of the relaxation of "multistate" systems, i.e., systems with more than two quantum states or two different chemical species, for linearized processes described by the "Master Equation" of the theory of transport processes. These results are compared with those obtained from the analysis of two-state relaxation processes and the concept of "relaxation time" is discussed in this framework. A discussion is presented of the transformation of microscopic to macroscopic relaxation equations. The existence of periodic and/or aperiodic oscillatory solution of the linear multistate relaxation equation is investigated. It is shown that the multistate relaxation equations admit of aperiodic oscillatory solutions.

Optical measurements on thin films of condensed gases at low temperatures, J. Kruger and W. J. Ambs, J. Opt. Soc. Am. 44, 1195 (1959).

A determination of the retractive indices of thin films of oxygen, nitrogen, carbon dioxide, water, argon, neon, and krypton condensed at $4.2^{\circ} \mathrm{K}$ using the $5461 \mathrm{~A}$ line of $\mathrm{Hg}$ was made with an ellipsometer. Similar measurements were also made on films of these gases condensed after passage through a microwave discharge. With the exception $\mathrm{O}_{2}, \mathrm{CO}_{2}$, and $\mathrm{H}_{2} \mathrm{O}$ no measurable differences in refractive indices were observed between films condensed from discharged and undischarged gases.

Invariant and complete stress functions for general continua, C. Truesdell, Arch. Rational Mech. Anal. 1, No. 4, 1 (1959).

This paper presents an organized survey of results, with proofs, concerning exact general solution of the underdetermined equations of motion of a continuous medium. Special attention is given to a flat space of $n$ dimensions, to an $n$-dimensional space of constant curvature, and to a 2 dimensional surface. Two methods are used: (1) the representation of a solenoidal vector as the curl of a vector potential, and (2) a variational formulation, essentially the converse of the principle of virtual work.

Wavelength definition of the meter, I. C. Gardner, Syst. of Units, Natl. and Intern. Aspects, Publ. Am. Assoc. Advance. Sci., No. 57, p. 53 (1959).

The different applications of the national prototype meter to the calibration of line standards, of end standards, and of wavelengths are presented. The advantages of a wavelength standard are considered and the progress of international conferences for redefining the meter in terms of a wavelength standard is set forth. One may expect final and conclusive action at the Conference (General) of Weights and Measures in 1960. The advantages to be anticipated from the development of an atomic beam source are discussed. 
Ethane carbon-carbon distance obtained from infrared spectra, H. C. Allen and E. K. Plyler, J. Chem. Phys. 31, No. 4, 1062 (1959).

The parallel band of ethane at $2753 \mathrm{~cm}^{-1}$ and the parallel band of ethane- $\mathrm{d}_{6}$ at $2816 \mathrm{~cm}^{-1}$ have been observed and analyzed. The resultant values of $B$ for $\mathrm{C}_{2} \mathrm{H}_{6}$ and $\mathrm{C}_{2} \mathrm{D}_{6}$ are $0.6637 \mathrm{~cm}^{-1}$ and $0.4598 \mathrm{~cm}^{-1}$, respectively. From these $B$ values the $\mathrm{C}-\mathrm{C}$ distance is found to be $1.534 \mathrm{~A}$ and the quantity

$\left(\gamma_{\mathrm{CC} / 2}+\gamma_{\mathrm{CH}} \cos \theta\right)^{2}+\gamma_{\mathrm{CH} / 2}{ }^{2} \sin ^{2} \theta=1.866 \mathrm{~A}$ where $\theta=180-\angle \mathrm{HCC}$.

If the $\gamma_{\mathrm{CH}}$ value of methane $(1.093 \mathrm{~A})$ is assumed, the $\angle \mathrm{HCC}$ is $109^{\circ} 45^{\prime}$

Young's modulus of various refractory materials as a function of temperature, J. B. Wachtman, Jr., and D. G. Lam, Jr., J. Am. Ceram. Soc. 42, No. 5, $254(1959)$.

Young's modulus as a function of temperature was determined by a dynamic method for single-crystal sapphire and ruby and for polycrystalline aluminum oxide, magnesium oxide, thorium oxide, mullite, spinel, stabilized zirconium oxide, silicon carbide, and nickel-bonded titanium carbide. For the single crystals, Young's modulus was found to decrease linearly with increasing temperature from $100^{\circ} \mathrm{C}$. to the highest temperature of measurement. For all the polycrystalline materials, except silicon carbide, stabilized zirconium oxide, and spinel, Young's modulus was found to decrease approximately linearly with increasing temperature until some temperature range characteristic of the material was reached in which Young's modulus decreased very rapidly and in a nonlinear manner with increasing temperature. This rapid decrease at high temperature is attributed to grainboundary slip. Stabilized zirconium oxide and spinel were found to have the same rapid decrease in Young's modulus at high temperature, but they also had a decidedly nonlinear temperature dependence at low temperature.

Branched-chain higher sugars. III. A 4-C-(hydroxymethyl)-pentose, R. Schaffer, J. Am. Chem. Soc. 81, 5452 (1959).

The applicability of a mixed aldol reaction for carbohydrate synthesis is demonstrated by the reaction of 5-aldo-1,2- 7 isopropylidene-D-xylo-pentofuranose (I) with formaldehyde. 4-C - (Hydroxymethyl) - 1,2 - $O$-isopropylidene - L-threo-pentofuranose (II) is isolated in $75 \%$ yield, and probably result: from reduction of the condensation product by a second molecule of formaldehyde through a crossed-Cannizzaro reaction. Hydrolysis of II gives the new branched-chain hexose. Oxidation of the sugar led to isolation of a crystalline calcium hexonate, and degradation of the latter yields previously unreported I-apiose. An alternative procedure involving $\mathrm{I}$ and formaldehyde, co-products of periodate oxidation of 1,2-O-isopropylidene-D-glucofuranose and used without intermediate crystallization of I, gives II in an overall yield of $58 \%$.

Vibrational spectrum of cyanate ion in various alkali halide lattices, A. Maki and J. C. Decius, J. Chem. Phys. 31, No. 3, 772 (1959).

The infrared absorption spectra of dilute solid solutions of cyanate ion in $\mathrm{KI}, \mathrm{KBr}, \mathrm{KCl}$, and $\mathrm{NaCl}$ single crystals have been examined between 600 and $5000 \mathrm{~cm}^{-1}$ at temperatures ranging from $150^{\circ}$ to $480^{\circ} \mathrm{K}$. Over fifty distinct maxima have been observed and assigned (including numerous isotopic, combination, and hot bands) in the most concentrated sample, for which $\mathrm{KBr}$ was the solvent. A strong Fermi resonance between $2 \omega_{2}$ and $\omega_{1}$ occurs. The vibrational energy has been expressed in terms of the eleven fundamental frequency and anharmonicity constants appropriate to a linear, unsymmetric triatomic molecule. Force constants in the KI solvent lattice are $f_{\mathrm{NC}}=15.51, f_{\mathrm{CO}}=11.03$, $f^{1}=1.35$, and $\left(f_{\mathrm{a}} / l_{1} l_{2}\right)=0.506 \mathrm{mdyne} / \mathrm{A}$, a result which shows very clearly that the $\mathrm{NC}$ bond is of higher order than the $\mathrm{CO}$ bond in the cyanate ion, and that the latter bond is notably weaker in cyanate ion than in $\mathrm{CO}_{2}$, where its force constant is 16.0 .

In general, the fundamental frequencies increase as the lattice constant decreases. Fairly detailed calculations show that although the induction energy term arising from the dipoles induced in the solvent by the vibrating cyanate ion is not negligible, the observed variation of frequency cannot be explained by the induction term alone and must involve the short-range, repulsive forces.

Use of crystal to display high energy X-ray images, J. S. Pruitt, Non-Destructive Testing XVII, No. 6, $359(1959)$

This paper describes an instrument composed of a large thallium activated sodium iodide crystal viewed by a television camera. An optical replica of the image produced by X-rays bombarding the crystal is displayed on a monitor. Evidence on the performance of this instrument, obtained both with radium $\gamma$-rays and 180 Mev X-rays, will be described. The image quality obtained with $\gamma$-rays is good enough for many radiographic purposes. At $180 \mathrm{Mev}$ where the quality is much poorer, the instrument has some value because of its ability to see gross detail continuously, even when looking through a thick absorber.

Thermodynamic properties of helium at low temperatures and high pressures, D. B. Mann and R. B. Stewart, J. Heat Transfer 81, 323 (1959).

The thermodynamic properties of helium have been compiled and correlated for a temperature range from 3.0 to $20^{\circ} \mathrm{K}$ for pressures to 100 atmospheres and for specific volumes from 5 to 800 liters per kilogram. The properties are presented on both the Temperature-Entropy and the Enthalpy-Entropy coordinate systems and include pressure, temperature, volume, entropy and enthalpy.

\section{Absolute photometry of the aurora - I. The ionized molecular nitrogen emission and the oxygen green line in the dark atmosphere, M. H. Rees, J. Atmos- pheric and Terrest. Phys. 14, 325 (1959).}

A low latitude aurora was measured photometrically. Three prominent emission features, $\lambda 3914$ band of 1. N.G. $\mathrm{N}_{2}{ }^{+}$, $\lambda 5577$ of $\mathrm{O} I$ and $\lambda 6300-6364$ of $\mathrm{O} I$, were isolated with narrow pass band filters and recorded continuously throughout the night in the meridian sweep. Absolute calibration was carried out, and corrections for the scattered light and atmospheric extinction were made.

The absolute emission intensity for $\lambda 3914$ and $\lambda 5577$ is given at a selected zenith distance. The ratio of instantaneous intensities $I(\mathrm{O}) 5577$ to $I(\mathrm{O}) 3914$ was found to be essentially constant over an intensity range of three powers of ten and to be independent of the type of auroral form.

The intensities and correlations obtained are consistent with the following excitation mechanisms. In the dark atmosphere the $\mathrm{N}_{2}{ }^{+}$is excited from ionizing collisions of incoming particles with atmospheric $\mathrm{N}_{2}$. It is concluded that the $\mathrm{OI}$ ג5577 line results from secondary electrons, arising from the primary ionization process above, colliding inelastically with oxygen atoms.

Absolute photometry of the aurora-II. Molecular nitrogen emission in the sunlit atmosphere, M. H. Rees, J. Atmospheric and Terrest. Phys. 14, 338 (1959).

The absolute emission intensity of a sunlit aurora was determined for the 1.N.G. $\lambda 3914$ band of $\mathrm{N}_{2}+$ by subtracting the emission intensity originating in the dark atmosphere from 
the total measured intensity. The method is based on the constancy of the ratio of emission intensity of the oxygen green line OI $\lambda 5577$ to the $\lambda 3914$ emission in the dark atmosphere. The ion density of $\mathrm{N}_{2}+$ is computed as a function of height from the emission rate per unit volume.

Atoms and free radicals by $\gamma$-irradiation at $4.2^{\circ} \mathrm{K}$, L. A. Wall, D. W. Brown, and R. E. Florin, J. Phys. Chem. 63, 1762 (1959).

In nitrogen, hydrogen, and deuterium at $4.2^{\circ} \mathrm{K}$ radiolysis produces atoms which are immobilized. They were identified by electron spin resonance (e.s.r.). In irradiated methane both hydrogen atoms and methyl radicals were found in approximately equal amounts. Repeated irradiation of the same samples in all cases led to more efficient production of active species. Added oxygen $(0.07 \%$ ) reduced the nitrogen atom yield of the initial run. Yields were studied as a function of irradiation dose. The maximum nitrogen atom concentration attained in solid nitrogen was $0.06 \%$, while in irradiated methane $0.15 \%$ of hydrogen atoms and $0.14 \%$ of methyl radicals were attained. Because of saturation of the e.s.r. spectra, the results with hydrogen and deuterium \& re low. High-energy radiation at low temperatures in general appears to be a useful and promising method for the production of stabilized free radicals or atoms.

Rotational transfer in the fluorescence spectrum of OH $\left({ }^{2} \Sigma^{+}\right)$, T. Carrington, J. Chem. Phys. 31, No. 5, 1418 (1959).

Fluorescence has been produced in $\mathrm{OH}$ molecules by absorption of the bismuth emission line $3067.7 \mathrm{~A}$, which excites a single rotational level in the ${ }^{2} \Sigma^{+}$electronic state. The relative population of this level and of other levels populated from it by collisional transfer are determined by intensity measurements on the fluorescence spectrum. The total probability of collisions producing transfer of rotational quanta from the eleventh level corresponds to a cross section roughly twice the kinetic theory value.

A note on the scattering of electrons from atomic hydrogen, A. Temkin, Phys. Rev. 116, No. 2, 358 (1959).

The distortion of atomic hydrogen by a slowly moving electron at a large distance from the center of the atom is examined. The problem is the initial phase of a previously described method for the calculation of electron scattering which takes this distortion into account. The initial (static) problem is solved analytically and extended to include higher order effects of the interaction of the electron with the atomic cloud. The construction of a wave function to describe scattering starting from the solution of the static problem is clarified. This yields as an incidental result a new approximation of the second order perturbation energy associated with the above distortion. A short discussion of the present experimental results for this scattering process is included.

Design of free-air ionization chambers for the soft X-ray region $(20-100 \mathbf{k v}), \mathrm{V}$. H. Ritz, Radiology 73, No. 6, 911 (1959).

Design criteria for standard free-air ionization chambers have been experimentally determined for the soft X-ray region. A tungsten target $X$-ray tube with a $2 \mathrm{~mm}$ beryllium window has been operated at exciting potentials of 20 to 100 kvep. Up to $4 \mathrm{~mm}$ of aluminum has been added to the inherent filtration of the tube.

The electric field distortion at the collecting electrode due to the proximity of the guard strip system has been studied as a function of the distance between the guard strips and the collecting electrode, the centerline to centerline distance between guard strips, and the thickness of the guard strips.

The radial distributions of the primary electron ionization and scattered photon induced ionization have been measured. Agreement with the work of Attix and DeLaVergne is good. Other correction factors of particular importance in the soft $\mathrm{X}$-ray region are discussed. The data indicate that a measurement of the exposure dose in roentgens can be made with a probable limit of error of $\pm 0.5 \%$ in this energy region.
Thermal conductivity of indium antimonide at low temperatures, E. V. Mielczarek and H. P. R. Frederikse, Phys. Rev. 115, 888 (1959).

Thermal conductivity measurements were made on a monocrystalline sample of indium antimonide from 10 to $50^{\circ} \mathrm{K}$. Umklapp, isotope, and boundary scattering contributions to the thermal resistivity were calculated from theoretical expressions and then subtracted from the measured value of thermal resistivity. The subsequent deduction of impurity scattering gives a value for the number of point impurities which is compatible with that given by electrical measurements.

Model for vibrational relaxation of diatomic gases behind shock waves, R. Herman and R. J. Rubin, Phys. of Fluids 2, No. 5, 547 (1959).

The problem of the vibrational relaxation of a system of harmonic oscillators is examined for the case in which the oscillators are in contact with a heat bath whose total heat capacity is finite and whose temperature therefore varies during the relaxation process. An analysis is carried out for processes in which the initial distribution of vibrational energy is a Boltzmann distribution. Application is made to the vibrational relaxation of a diatomic gas behind a shock wave.

The dissociation constant of $\mathrm{CaOH} \pm$ from $0^{\circ}$ to $40^{\circ}$ C, R. G. Bates, V. E. Bower, R. G. Canham, and J. E. Prue, Trans. Faraday Soc. 55, No. 444, 2062 (1959).

An attempt has been made to derive the dissociation constant of the base $\mathrm{CaOH}$ at eight temperatures from $0^{\circ}$ to $40^{\circ} \mathrm{C}$ from e.m.f. data for cells without liquid junction composed of hydrogen and silver + silver chloride electrodes. Nineteen of the 27 solutions studied contained calcium hydroxide and potassium chloride and the remainder contained calcium hydroxide and calcium chloride. The dissociation constant $K_{2}$ obtained from the experimental data is strongly influenced by the arbitrary choice of $\gamma \mathrm{Cl} / \gamma \mathrm{OH}$ necessary to evaluate the molality of hydroxide ion in the solutions. If the activity coefficients of these two ions are assumed to be equal at ionic strengths up to $0.08, K_{2}$ is found to be 0.071 mole $\mathrm{kg}^{-1}$ at $25^{\circ} \mathrm{C}$; if $\log (\gamma \mathrm{Cl} / \gamma \mathrm{OH})=-0.31$ is assumed, a value of 0.054 mole $\mathrm{kg}^{-1}$ is obtained. Similar uncertainties are inherent in earlier determinations of this constant by solubility, e.m.f., and kinetic methods.

\section{Other NBS Publications}

\section{Journal of Research, Section 64C, No. 2, April-June 1960. 75 cents.}

Measurement of cobalt-60 and cesium-137 gamma rays with a free-air chamber. H. O. Wyckoff. (See above abstracts.) Apparatus for the measurement of the normal spectral emissivity in the infrared. Arthur G. Maki, Ralph Stair, and Russell G. Johnston.

Electrostatic deflection plates for cathode-ray tubes. I. Design of single-bend deflection plates with parallel entrance sections. II. Deflection defocusing distortion of singlebend deflection plates with parallel entrance sections. Lothar Frenkel.

The functional synthesis of linear plots. J. P. Vinti and R. F. Dressler.

Radiation field from a rectangular source. J. H. Hubbell, R. L. Bach, and J. C. Lamkin. (See above abstracts.)

Microwave attenuation measurements with accuracies from 0.0001 to 0.06 decibel over a range of 0.01 to 50 decibels. G. F. Engen and R. W. Beatty.

Effect of oleophobic films on metal fatigue. H. E. Frankel, J. A. Bennett, and W. L. Holshouser.

Ratio-recording spectroradiometer. Harry K. Hammond III, Warren L. Holford, and Milton L. Kuder.

An intermittent-action camera with absolute time calibration. G. Hefley, R. H. Doherty, and E. L. Berger. 
Journal of Research, Section 64D, No. 3, May-June 1960. 70 cents.

A theory of radar scattering by the moon. T. B. A. Senior and K. M. Siegel.

A theory of wavelength dependence in ultrahigh frequency transhorizon propagation based on meteorological considerations. Ralph Bolgiano, Jr.

A preliminary study of radiometeorological effects on beyondhorizon propagation. F. Ikegami.

Trade-wind inversion as a transoceanic duct. M. Katzin, H. Pezzner, B. Y.-C. Koo, J. V. Larson, and J. C. Katzin. An analysis of propagation measurements made at 418 megacycles per second well beyond the radio horizon (a digest). H. B. Janes, J. C. Stroud, and M. T. Decker.

On the calculation of the departures of radio wave bending from normal. B. R. Bean and E. J. Dutton.

On the mode theory of very-low-frequency propagation in the presence of a transverse magnetic field. D. D. Crombie.

On the theory of reflection of low- and very-low-radiofrequency waves from the ionosphere. J. Ralph Johler and Lillie C. Walters.

Focusing, defocusing, and refraction in a circularly stratified atmosphere. K. Toman.

Response of a loaded electric dipole in an imperfectly conducting cylinder of finite length. Charles W. Harrison, Jr., and Ronold W. P. King. (See above abstracts.)

Impedance characteristics of a uniform current loop having a spherical core. Saburo Adachi.

Specification for dry cells and batteries (supersedes Circ. 559). NBS Handb. 71, (1959) 25 cents.

Report of the 44th National Conference on Weights and Measures 1959. NBS Misc. Publ. 228, (1959) 65 cents.

Research Highlights of the National Bureau of Standards Annual Report, Fiscal Year 1959. NBS Misc. Publ. 229 (1959) 55 cents.

An atlas of oblique-incidence ionograms, V. Agy, K. Davies, and R. Salaman, NBS Tech. Note 31 (PB151390) (1959) $\$ 2.25$.

Service area of an airborne television station, M. T. Decker, NBS Tech. Note 35 (PB151394) (1959) 75 cents.

The following are published in Proc. Intern. Rubber Conf., Nov. 8 to 13, 1959 (Washington, D.C., 1959):

Power loss and operating temperature of tires, R. D. Stiehler, M. N. Steel, G. G. Richey, J. Mandel, and R. H. Hobbs, p. 73.

An indoor tester for measuring tire tread wear, G. G. Richey, J. Mandel, and R. D. Stiehler, p. 104.

Measurement of the aging of rubber vulcanizates, J. Mandel, F. L. Roth, M. N. Steel, and R. D. Stiehler, p. 221.

Standard materials for rubber compounding, F. L. Roth and R. D. Stiehler, p. 232.

On the convergence of Gauss' alternating procedure in the method of least squares, A. M. Ostrowski, Ann. Mat. Pura Appl. (IV) (Bologna, Italy) 48, 229 (1959).

The anomalous inversion in cristobalite, R. F. Walker, The kinetics of high temperature processes, 228 pages (J. Wiley \& Sons, New York, N.Y., 1959).

Digital recording of electrocardiographic data for analysis by a digital computer, L. Taback, E. Marden, H. L. Mason, and H. V. Pipberger, IRE Trans. Med. Electron. ME-6. 167 (1959).

Determination of starch in paper. A Comparison of the TAPPI enzymatic, and spectrophotometric methods, J. L. Harvey, B. W. Forshee, and D. G. Fletcher, Tappi 42, No. 11, 878 (1959).

Glass research at the National Bureau of Standards, C. H. Hahner, Glass Ind., p. 588 (1959).

Applications of the molecular refractivity in radio metrology, B. R. Bean and R. M. Gallet, J. Geophys. Research 64, No. 10, 1439 (1959).

The measuring process, J. Mandel, Technometrics 1, No. 3, 251 (1959).

Fire research at the National Bureau of Standards, A. F. Robertson, Fire Research Abstr. Rev. I, No. 4, 159 (1959).

Refractive indices and transmittances of several optical glasses in the infrared, G. W. Cleek, J. J. Villa, and C. H. Hahner, J. Opt. Soc. Am. 49, No. 11, 1090 (1959).
Preliminary assessment of the IGY, A. H. Shapley, Proc. Natl. Electron. Conf., p. 1 (1958).

Factorial experiments in life testing, M. Zelen, Technometrics 1, No. 3, 269 (1959).

Direction findings on whistlers, J. M. Watts, J. Geophrs. Research 64, No. 11, 2029 (1959)

The construction of Hadamard matrices, E. C. Dade and K. Goldberg, Mich. Math. J. 6, 247 (1959).

Geomagnetic and ionospheric phenomena associated with nuclear explosions, S. Matsushita, Nature 184, BA33 (1959.)

Stress corrosion cracking in low carbon steel, H. L. Logan, Proc. Phys. Met. Stress Corrosion Fracture Conf., Pittsburgh, Pa., Apr. 2-3, 1959, reprinted from Met. Soc. Conf., p. 295 (Interscience Publishers, New York, N.Y., 1959).

Higher oxides of silver, H. E. Swanson, J. Electrochem. Soc. 106, No. 12, 1073 (1959).

Oscillatory behavior of magnetic susceptibility and electronic conductivity, A. H. Kahn and H. P. R. Frederikse, vol. IX, Adv. Solid State Phys., p. 257 (Academic Press Inc., New York, N.Y., 1959).

Geomagnetic activity following large solar flares, C. S. Warwick and R. T. Hansen, J. Atmospheric and Terrest. Phys. 14, 287 (1959).

Bending and stretching of corrugated diaphragms, R. F. Dressler, ASME Trans. 81D, No. 4, 651 (1959).

Simplification of systems of units currently evolving in the electrical field, F. B. Silsbee, Systems of Units, Am. Assoc. for Advance. Sci., p. 7 (1959).

Determination of viscosity, J. F. Swindells, ch. XII, Phys. Methods of Organic Chem., pt. I, p. 689 (Interscience Publishers Inc., New York, N.Y., 1959).

Triaxial tension at the head of a rapidly running crack in a plate, J. M. Frankland, Trans. ASME [E] 26, No. 4, 570 (1959).

Poly (tetrafluoroethylene) - a radiation-resistant polymer, L. A. Wall and R. E. Florin, letter to ed., J. Appl. Polymer Sci. II, No. 5, 251 (1959).

On the approximate daytime constancy of the absorption of radio waves in the lower ionosphere, $\mathrm{S}$. Chapman and $\mathrm{K}$. Davies, J. Atmospheric and Terrest. Phys. 13, No. 1-2, 86 (1958).

An X-ray crystallographic study of Schroeckingerite and its dehydration product, D. K. Smith, Am. Mineralogist 44, 1020 (1959).

Modular forms whose coefficients possess multiplicative properties, M. Newman, Ann. Math. 70, 478 (1959).

The analysis of latin squares with a certain type of rowcolumn interaction, J. Mandel, Technometries 1, No. 4, 379 (1959).

A note on algebras, A. J. Goldman, Am. Math. Mo. 66, 795 (1959).

Transistor $\mathrm{P}-\mathrm{A}$ amplifier, G. F. Montgomery and F. R. Bretemps, Electronic Ind. 19, No. 1, 196 (1960).

Linear differential equations of the second order with a large parameter, F. W. J. Olver, J. Soc. Indust. Appl. Math. 7, 306 (1959).

Sorting devices in postal tests. 1: Proposal for an electromagnetic sorter, S. Henig, Automatic Control 11, No. 6, 6 (1959).

Evaluation of chemical analyses on two rocks, W. J. Youden, Technometrics 1, No. 4, 409 (1959).

Nuclear electronics, L. Costrell, Nucleonics Mag. 18, No. 1, 124 (1960).

Microscopical studies of failure in polymers, S. B. Newman, ASTM Spec. Tech. Pub. 257, 132 (1959).

Flare-associated bursts at $18 \mathrm{Mc} / \mathrm{s}$, C. Warwick and J. W. Warwick, Paris Symp. on Radio Astron., p. 203 (IAU Symp. No. 9 and URSI Symp. No. 1, 1959).

* Publications for which a price is indicated (except for NBS Technical Notes) are available only from the Superintendent of Documents, U.S. Government Printing Office, Washington 25, D.C. (foreign postage, one-fourth additional). Technical Notes are available only from the Office of Technical Services, U.S. Department of Commerce, Washington 25, D.C. (order by PB number). Reprints from outside journals and the NBS Journal of Research may often be obtained directly from the author. 